\title{
Evolution of the resonance and incommensurate spin fluctuations in superconducting $\mathrm{YBa}_{2} \mathrm{Cu}_{3} \mathrm{O}_{6+x}$
}

\author{
Pengcheng Dai, ${ }^{1}$ 月 H. A. Mook, ${ }^{1}$ R. D. Hunt, ${ }^{2}$ and F. Doğan ${ }^{3}$ \\ ${ }^{1}$ Solid State Division, Oak Ridge National Laboratory, Oak Ridge, Tennessee 37831-6393 \\ ${ }^{2}$ Chemical Technology Division, Oak Ridge National Laboratory, Oak Ridge, Tennessee 37831-6221 \\ ${ }^{3}$ Department of Materials Science and Engineering, \\ University of Washington, Seattle, Washington 98195
}

(Dated: October 23, 2018)

\begin{abstract}
Polarized and unpolarized neutron triple-axis spectrometry was used to study the dynamical magnetic susceptibility $\chi^{\prime \prime}(\mathbf{q}, \omega)$ as a function of energy $(\hbar \omega)$ and wave vector $(\mathbf{q})$ in a wide temperature range for the bilayer superconductor $\mathrm{YBa}_{2} \mathrm{Cu}_{3} \mathrm{O}_{6+x}$ with oxygen concentrations, $x$, of $0.45,0.5$, $0.6,0.7,0.8,0.93$, and 0.95 . The most prominent features in the magnetic spectra include a spin gap in the superconducting state, a pseudogap in the normal state, the much-discussed resonance, and incommensurate spin fluctuations below the resonance. We establish the doping dependence of the spin gap in the superconducting state, the resonance energy, and the incommensurability of the spin fluctuations. The magnitude of the spin gap $\left(E_{s g}\right)$ up to the optimal doping is proportional to the superconducting transition temperature $T_{c}$ with $E_{s g} / k_{B} T_{c}=3.8$. The resonance, which exists exclusively below $T_{c}$ for highly doped $\mathrm{YBa}_{2} \mathrm{Cu}_{3} \mathrm{O}_{6+x}$ with $x=0.93$ and 0.95 , appears above $T_{c}$ for underdoped compounds with $x \leq 0.8$. The resonance energy $\left(E_{r}\right)$ also scales with $k_{B} T_{c}$, but saturates at $E_{r} \approx 40 \mathrm{meV}$ for $x$ close to 0.93 . The incommensurate spin fluctuations at energies below the resonance have structures similar to that of the single-layer superconducting $\mathrm{La}_{2-x} \mathrm{Sr}_{x} \mathrm{CuO}_{4}$. However, there are also important differences. While the incommensurability $(\delta)$ of the spin fluctuations in $\mathrm{La}_{2-x} \mathrm{Sr}_{x} \mathrm{CuO}_{4}$ is proportional to $T_{c}$ for the entire hole-doping range up to the optimal value, the incommensurability in $\mathrm{YBa}_{2} \mathrm{Cu}_{3} \mathrm{O}_{6+x}$ increases with $T_{c}$ for low oxygen doping and saturates to $\delta=0.1$ for $x \geq 0.6$. In addition, the incommensurability decreases with increasing energy close to the resonance. Finally, the incommensurate spin fluctuations appear above $T_{c}$ in underdoped compounds with $x \leq 0.6$ but for highly doped materials they are only observed below $T_{c}$. We discuss in detail the procedure used for separating the magnetic scattering from phonon and other spurious effects. In the comparison of our experimental results with various microscopic theoretical models, particular emphasis was made to address the similarities and differences in the spin fluctuations of the two most studied superconductors. Finally, we briefly mention recent magnetic field dependent studies of the spin fluctuations and discuss their relevance in understanding the microscopic origin of the resonance.
\end{abstract}

\section{INTRODUCTION}

The parent compounds of the high-transitiontemperature (high- $T_{c}$ ) copper-oxide superconductors are antiferromagnetic (AF) insulators characterized by a simple doubling of the crystallographic unit cell in the $\mathrm{CuO}_{2}$ planes [1, 2]. When holes are doped into these planes, the long-range AF-ordered phase is destroyed and the lamellar copper-oxide materials become metallic and superconducting. This raises the fundamental question of the role of the magnetism in the superconductivity of these materials. Over the past decade, experimental probes such as nuclear magnetic resonance (NMR), muon spin resonance $(\mu \mathrm{SR})$, and neutron scattering have been used to address this fundamental question. In particular, neutron scattering experiments have unambiguously shown the presence of short-range AF spin correlations (fluctuations) in cuprate superconductors such as $\mathrm{La}_{2-x} \mathrm{Sr}_{x} \mathrm{CuO}_{4}$ 3, 4, 5, 6, 7, 8, 9, 10, 11] and $\mathrm{YBa}_{2} \mathrm{Cu}_{3} \mathrm{O}_{6+x}$ [12, 13, 14, 15, 16, 17, 18, 19, 20, 21, 22, 23, 24, 25, 26, 27, at all

*Electronic address: daip@ornl.gov doping levels, $x$. Even so, the role of such fluctuations in the pairing mechanism and superconductivity is still a subject of controversy [28].

If spin fluctuations are important for the mechanism of high- $T_{c}$ superconductivity, they should be universal for all copper oxide systems. For the $\mathrm{La}_{2} \mathrm{CuO}_{4}$ family of materials, which has a single $\mathrm{CuO}_{2}$ layer per unit cell, superconductivity can be induced by the substitution of $\mathrm{Sr}, \mathrm{Ba}$, or $\mathrm{Ca}$ for La or by intercalation of excess oxygen. The low-energy spin fluctuations have been observed at a quartet of incommensurate wave vectors (Fig. 1) away from the AF Bragg positions, often referred to as $q_{0}=(\pi, \pi)$, in the $x=0$ compounds [11]. These modulated low-frequency spin fluctuations persist in both the normal and superconducting states with a suppression of their intensity below $T_{c}[9,10]$. As a function of the doping, the incommensurability $\delta$ increases linearly with $x$ until saturating at $\delta \simeq 1 / 8$ for $x \geq 1 / 8$ [5, 8, 29. Furthermore, the incommensurability depends only on the doping level and the unbound charge introduced by the dopants, and not on the method for introducing the charge 11. As a function of increasing frequency, the incommensurability does not change [6], and no particularly sharp features have been identified [11]. 


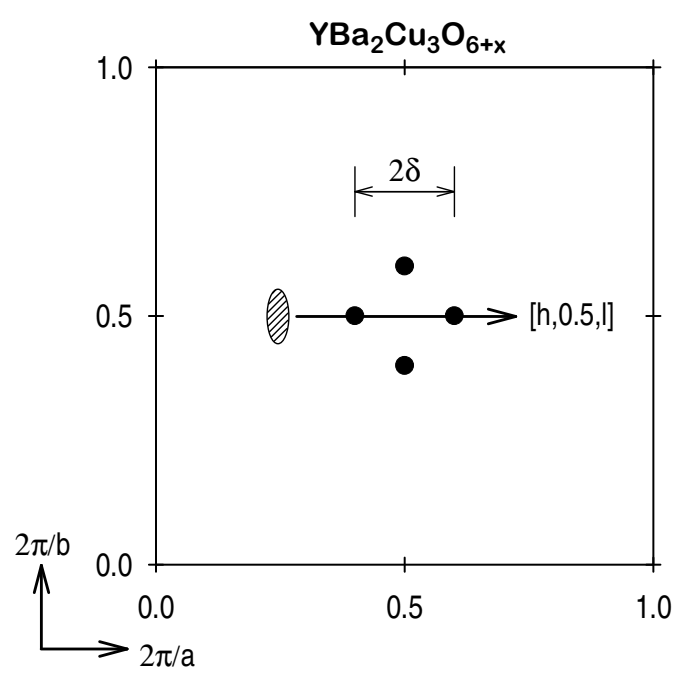

FIG. 1: The reciprocal space diagram and scattering geometry used in the experiments. Note that the scan direction for most of the measurements is along $[h, 0.5, l]$. The structure of the incommensurate spin fluctuations is very similar to that of $\mathrm{La}_{2-x} \mathrm{Sr}_{x} \mathrm{CuO}_{4}$ as shown by Mook et al. [24]. In this article, we define the separation between the incommensurfigure 1 peaks as $2 \delta$ as shown in the figure. The shaded ellipse shows that the narrow part of the resolution volume is along the scan direction.

For $\mathrm{YBa}_{2} \mathrm{Cu}_{3} \mathrm{O}_{6+x}$, which has two $\mathrm{CuO}_{2}$ planes per unit cell (bilayer), the situation is more complex. Early measurements [12, 14, 16, 17, 18] have shown that the most prominent feature in the spin fluctuations spectra in the highly doped $\mathrm{YBa}_{2} \mathrm{Cu}_{3} \mathrm{O}_{6+x}(x \geq 0.9)$ is a sharp peak which appears below $T_{c}$ at an energy of $\sim 41 \mathrm{meV}$. When scanned at fixed energy as a function of wave vector, the sharp peak is centered at $(\pi, \pi)$ and generally referred to as a resonance. For underdoped $\mathrm{YBa}_{2} \mathrm{Cu}_{3} \mathrm{O}_{6+x}$, the resonance also peaks at $(\pi, \pi)$ but with a reduced energy 19, 20, 21]. In $\mathrm{YBa}_{2} \mathrm{Cu}_{3} \mathrm{O}_{6.6}$, it occurs at $34 \mathrm{meV}$ and is superposed on a continuum which is gapped at low energies [27]. Below the resonance frequency, the continuum is actually strongest at a quartet of incommensurate positions consistent with those in $\mathrm{La}_{2-x} \mathrm{Sr}_{x} \mathrm{CuO}_{4}$ of the same hole doping (Fig. 1) 22, 23, 24]. This discovery along with similar observations in $\mathrm{YBa}_{2} \mathrm{Cu}_{3} \mathrm{O}_{6.7}$ [25] suggests that incommensurate spin fluctuations may be universal to all high- $T_{c}$ superconductors.

In this article, we report systematic unpolarized and polarized inelastic neutron scattering studies of spin fluctuations in $\mathrm{YBa}_{2} \mathrm{Cu}_{3} \mathrm{O}_{6+x}$. By carefully separating the magnetic signal from phonons and other spurious effects, we determine the wave vector and energy dependence of the spin dynamical susceptibility, $\chi^{\prime \prime}(\mathbf{q}, \omega)$, as a function of temperature and doping concentration. For $\mathrm{YBa}_{2} \mathrm{Cu}_{3} \mathrm{O}_{6+x}$ with oxygen concentrations at all doping levels addressed in this article, we observe a spin gap in the superconducting state, a commensurate resonance, and incommensurate spin fluctuations at energies below the resonance. The magnitude of the spin gap in the superconducting state $\left(E_{s g}\right)$ is proportional to $k_{B} T_{c}$ with $E_{s g} / k_{B} T_{c}=3.8$. The resonance appears above $T_{c}$ for underdoped compounds but occurs exclusively below $T_{c}$ for optimal and overdoped materials. The incommensurability of the excitations below the resonance decreases with increasing energy until merging into the resonance. For $\mathrm{YBa}_{2} \mathrm{Cu}_{3} \mathrm{O}_{6+x}$ with $x \leq 0.6$, the incommensurability is approximately proportional to $T_{c}$. For oxygen concentrations $x$ above 0.6 , the incommensurability is independent of hole-doping and saturates at $\delta=1 / 10$. In the case of $\mathrm{YBa}_{2} \mathrm{Cu}_{3} \mathrm{O}_{6.8}$, the commensurate low-frequency normal state magnetic excitations centered around $(\pi, \pi)$ are suppressed with the opening of a large spin gap in the superconducting state. For $\mathrm{YBa}_{2} \mathrm{Cu}_{3} \mathrm{O}_{6.93}$ and $\mathrm{YBa}_{2} \mathrm{Cu}_{3} \mathrm{O}_{6.95}$, the normal state spin fluctuations are below the detection limit of current triple-axis spectroscopy, and the magnetic fluctuations in the superconducting state are dominated by the well-discussed resonance at $\sim 40 \mathrm{meV}$ [12, 14, 16, 17, 18] and incommensurate spin fluctuations below the resonance.

The rest of the paper is organized as follows. The next section describes the experimental procedures, including details of sample characterization and spectrometer setup. In Sec. IIIA, polarized and unpolarized neutron scattering data for $\mathrm{YBa}_{2} \mathrm{Cu}_{3} \mathrm{O}_{6+x}$ with oxygen concentrations, $x$, of $\sim 0.45,0.5,0.6,0.7,0.8,0.93$, and 0.95 are presented with a detailed description of the procedures used to extract magnetic scattering. In Sec. IIIB, a comparison of the results with those for $\mathrm{La}_{2-x} \mathrm{Sr}_{x} \mathrm{CuO}_{4}$ is presented. The issues of the doping dependence of the incommensurability, the spin gap in the superconducting state, the resonance in the normal state of underdoped materials, and resonance energy are addressed. Finally, the results presented in this paper and recent magnetic field dependence measurements of the spin fluctuations are discussed with the predictions and consequences of various theoretical models. Section IV gives a brief summary of the major conclusions of this work.

\section{EXPERIMENTAL DETAILS}

\section{A. Sample characterization}

The experiments were performed on large twined crystals of $\mathrm{YBa}_{2} \mathrm{Cu}_{3} \mathrm{O}_{6+x}$ that were prepared by the meltgrown technique at the University of Washington. As emphasized in previous publications [14, 19], these samples contain considerable amounts of $\mathrm{Y}_{2} \mathrm{BaCuO}_{5}$ [30] as an impurity. The structural and superconducting properties of these melt-grown $\mathrm{YBa}_{2} \mathrm{Cu}_{3} \mathrm{O}_{6+x}$ samples with different $\mathrm{Y}_{2} \mathrm{BaCuO}_{5}$ concentrations have been studied in detail by Gopalan et al. [31]. These authors found that the $\mathrm{YBa}_{2} \mathrm{Cu}_{3} \mathrm{O}_{6+x}$ platelet width and crack width decrease with increasing $\mathrm{Y}_{2} \mathrm{BaCuO}_{5}$ content. The reduced average $\mathrm{YBa}_{2} \mathrm{Cu}_{3} \mathrm{O}_{6+x}$ grain width may facilitate the forma- 
tion of equilibrium crystal structures at various oxygen concentrations. In a recent study of oxygen equilibration and chemical diffusion in massive $\mathrm{YBa}_{2} \mathrm{Cu}_{3} \mathrm{O}_{6+x}$ crystals, Lindemer [32] found that melt-grown samples containing a significant amount of $\mathrm{Y}_{2} \mathrm{BaCuO}_{5}$ reached their equilibrium values of oxygen concentration in a dynamic flow of oxygen considerably faster than samples without this impurity. These results are consistent with the notion that oxygen invariant $\mathrm{Y}_{2} \mathrm{BaCuO}_{5}$, micro-cracks, and the boundaries between twin grains are important in providing oxygen pathways through these large samples of $\mathrm{YBa}_{2} \mathrm{Cu}_{3} \mathrm{O}_{6+x}$.

For nonstoichiometric pure phase $\mathrm{YBa}_{2} \mathrm{Cu}_{3} \mathrm{O}_{6+x}$, Lindemer and coworkers [33] have established the thermodynamic equilibrium phase diagram of oxygen concentration versus temperature and oxygen pressure $(T-p)$. We oxygenate $\mathrm{YBa}_{2} \mathrm{Cu}_{3} \mathrm{O}_{6+x}$ in a vacuum thermogravimetric apparatus (TGA) by annealing to the equilibrium weight $( \pm 0.1 \mathrm{mg})$, equivalent to a sensitivity in $6+x$ of 0.0003 per mol, at $T-p\left[\mathrm{O}_{2}\right]$ conditions for the desired oxygen doping following previously established models [33]. Instead of quenching the sample after annealing, the partial pressure of oxygen in the TGA was adjusted during the cooling process to maintain a constant sample mass, thus ensuring uniformity in oxygen content and an equilibrium crystal structure. Seven single crystals of $\mathrm{YBa}_{2} \mathrm{Cu}_{3} \mathrm{O}_{6+x}$ were oxygenated in this way to obtain $x$ of $0.45,0.5,0.6,0.7,0.8,0.93$, and 0.95 .

The superconducting properties of these $\mathrm{YBa}_{2} \mathrm{Cu}_{3} \mathrm{O}_{6+x}$ crystals were characterized by a SQUID magnetometer. Susceptibility measurements made on small pieces cut from these samples show superconducting transition temperatures of $\sim 48 \pm 6 \mathrm{~K}$, $52 \pm 3 \mathrm{~K}, 62.7 \pm 2.7 \mathrm{~K}, 74 \pm 2.7 \mathrm{~K}, 82 \pm 1.25 \mathrm{~K}$, and $92.5 \pm 0.5 \mathrm{~K}$, for $\mathrm{YBa}_{2} \mathrm{Cu}_{3} \mathrm{O}_{6.45}, \mathrm{YBa}_{2} \mathrm{Cu}_{3} \mathrm{O}_{6.5}$, $\mathrm{YBa}_{2} \mathrm{Cu}_{3} \mathrm{O}_{6.6}, \quad \mathrm{YBa}_{2} \mathrm{Cu}_{3} \mathrm{O}_{6.7}, \quad \mathrm{YBa}_{2} \mathrm{Cu}_{3} \mathrm{O}_{6.8}, \quad$ and $\mathrm{YBa}_{2} \mathrm{Cu}_{3} \mathrm{O}_{6.93}$, respectively. The transition temperature for $\mathrm{YBa}_{2} \mathrm{Cu}_{3} \mathrm{O}_{6.95}$ of $92 \mathrm{~K}$ is estimated from the temperature dependence of the resonance [14, 27]. Although the widths of superconducting transition temperatures for $\mathrm{YBa}_{2} \mathrm{Cu}_{3} \mathrm{O}_{6+x}$ with $x \geq 0.6$ are usually sharp $(<3$ $\mathrm{K})$, the transition widths for lower doping samples are considerably broader. This appears to be the intrinsic property of the underdoped $\mathrm{YBa}_{2} \mathrm{Cu}_{3} \mathrm{O}_{6+x}$ [33].

To quantitatively determine the effectiveness of this oxygenation technique and the composition of the meltgrown crystal, two pieces were cut from a 96-g sample of $\mathrm{YBa}_{2} \mathrm{Cu}_{3} \mathrm{O}_{6.93}$. Sample $A$ weighed approximately $15-\mathrm{g}$ and was crushed into fine powder. Sample $B$ weighed 14.98-g. Powder diffraction measurements were performed on sample $A$ at room temperature using the HB4 high-resolution powder diffractometer with an incident beam wavelength of $1.0314 \AA$ at the High-Flux Isotope Reactor (HFIR) at Oak Ridge National Laboratory [34. Rietveld analysis of the powder data $\left(R_{w p}=6.55 \%\right.$, $\left.\chi^{2}=1.447\right)$ revealed that the sample contains 83.6 mol\% $\mathrm{YBa}_{2} \mathrm{Cu}_{3} \mathrm{O}_{6+x}$ with the partial oxygen occupancies $[\mathrm{O}(1)]$ at $0.96 \pm 3$ and $16.4 \mathrm{~mol} \% \mathrm{Y}_{2} \mathrm{BaCuO}_{5}$. The lattice parameters for the $\mathrm{YBa}_{2} \mathrm{Cu}_{3} \mathrm{O}_{6.93}[a=3.8188(2)$ $\AA, b=3.8856(2) \AA$, and $c=11.6920(5) \AA]$ are slightly different from that of the $\mathrm{YBa}_{2} \mathrm{Cu}_{3} \mathrm{O}_{6.93}$ sample reported by Jorgensen et al. [35], but they agree well with those of the $\mathrm{YBa}_{2} \mathrm{Cu}_{3} \mathrm{O}_{6+x}(0.93 \leq x<1)$ crystals of Altendorf et al. [36].

To further verify the partial oxygen content, sample $B$ was subjected to independent thermogravimetric analysis. The 14.98 -g specimen $(B)$ was subjected to the deoxygenation study in the TGA. The weight change of $B$ relative to the room-temperature weight was measured after equilibration at $573 \mathrm{~K}$ and at successively higher temperatures. The results were converted to $6+x$ values and compared to the expected behavior [33]. This analysis demonstrated that the initial oxygen content of $B$ was $6.93 \pm 0.005$, thus confirming the effectiveness of our oxygenation technique.

In an unpublished work, Chakoumakos and Lindemer [37] have systematically studied the lattice parameters of the powder $\mathrm{YBa}_{2} \mathrm{Cu}_{3} \mathrm{O}_{6+x}$ oxygenated using this technique. They performed Rietveld analysis of the powder neutron diffraction data obtained on HB-4 for a series $\mathrm{YBa}_{2} \mathrm{Cu}_{3} \mathrm{O}_{6+x}$ samples and established the relationship between oxygen concentration and lattice parameters (Fig. 2). High resolution measurements on the single crystals oxygenated in our study indicated that the lattice parameters have virtually the same value as powders. As a consequence, we used the lattice parameters established in their powder work for our single-crystal inelastic neutron scattering measurements.

\section{B. Neutron scattering}

Inelastic neutron scattering measurements were made at HFIR using the HB-1 and HB-3 triple-axis spectrometers. The momentum transfer $\left(q_{x}, q_{y}, q_{z}\right)$ is measured in units of $\AA^{-1}$ and reciprocal space positions are specified in reciprocal lattice units ( rlu) $(h, k, l)=$ $\left(q_{x} a / 2 \pi, q_{y} b / 2 \pi, q_{z} c / 2 \pi\right)$, where $a, b(\approx a)$, and $c$ are the lattice parameters of the orthorhombic unit cell of $\mathrm{YBa}_{2} \mathrm{Cu}_{3} \mathrm{O}_{6+x}$ (see Figs. 1 and 2). The magnetic neutron scattering directly measures the imaginary part of the generalized spin susceptibility $\chi^{\prime \prime}(\mathbf{q}, \omega)$ for momentum transfer $\mathbf{q}=\mathbf{k}_{i}-\mathbf{k}_{f}$ and energy transfer $\hbar \omega$, where $\mathbf{k}_{i}$ and $\mathbf{k}_{f}$ are the incident and final neutron wave vectors, respectively. The scattering cross-section for an isotropic system [38] is

$$
\frac{\mathrm{d}^{2} \sigma}{\mathrm{d} \Omega \mathrm{d} E}=\frac{2}{\pi g^{2}} \frac{k_{f}}{k_{i}} r_{0}^{2}|F(\mathbf{q})|^{2} \frac{1}{1-\exp \left(-\hbar \omega / k_{B} T\right)} \chi^{\prime \prime}(\mathbf{q}, \omega) / \mu_{\mathrm{B}}^{2}
$$

where $g$ is the Lande factor $(\approx 2), r_{0}$ is $5.4 \times 10^{-13}$ $\mathrm{cm},|F(\mathbf{q})|$ is the magnetic form factor, $[n(\omega)+1]=$ $1 /\left[1-\exp \left(-\hbar \omega / k_{B} T\right)\right]$ is the Bose population factor, and $\mu_{B}$ is the Bohr magneton. The major difficulty in studying spin fluctuations in high- $T_{c}$ materials is to separate the magnetic scattering from (single- and multi-) 


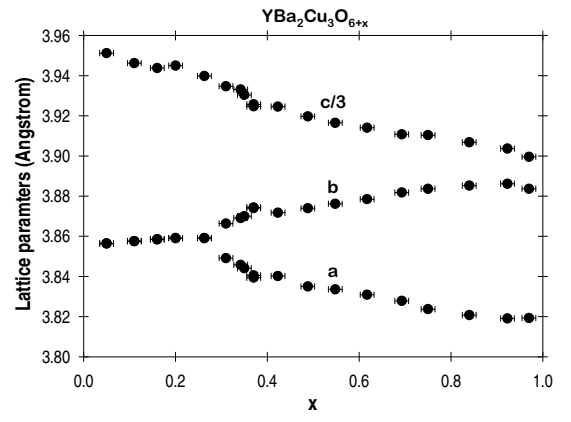

Figure 2
Dai et al.

FIG. 2: Lattice parameters of $\mathrm{YBa}_{2} \mathrm{Cu}_{3} \mathrm{O}_{6+x}$ as a function of oxygen concentration $x$ from Chakoumakos and Lindemer [37]. The results are obtained using a high-resolution powder diffractometer on powder samples oxygenated in the same way as the single crystals in this work. The vertical error bars are smaller than the size of the symbols.

phonon scattering and other spurious processes. While many spurious events such as accidental Bragg scattering can be identified by performing the desired inelastic scan in the two-axis mode 13], two approaches are used to separate magnetic from phonon scattering.

We first describe the use of neutron polarization analysis [39] which, in principle, can unambiguously separate magnetic from non magnetic processes. For the polarized experimental setup, a crystal of ${ }^{57} \mathrm{Fe}(\mathrm{Si})[d(110)=2.0202$ $\AA]$ and crystals of Heusler alloy $\mathrm{Cu}_{2} \mathrm{MnAl}[d(111)=$ $3.4641 \AA]$ were used as a monochromator and analyzer, respectively. The polarization state of the neutron beam can be changed by a spin-flipping device (Mezei coil) that is placed in the incident beam before the sample. The combination of a ${ }^{57} \mathrm{Fe}(\mathrm{Si})$ (110) monochromator and a Heusler (111) analyzer permits spin-flip (SF) measurements without using the Mezei coil because the polarization of the neutrons scattered by the ${ }^{57} \mathrm{Fe}(\mathrm{Si})$ is along the applied field direction while it is opposite for the Heusler crystal. For the measurements, the final neutron energy was fixed at $30.5 \mathrm{meV}$, and a pyrolytic graphite $(\mathrm{PG})$ filter was used before the analyzer. The polarization is usually determined by measuring the flipping ratio $R$, defined as the ratio of neutrons reflected by the analyzer with the flipper (Mezei coil) "on" [which corresponds to neutron nonspin flip (NSF), or ++ ] and "off" [neutron spin-flip (SF) or +-]. Assuming the flipping ratios for guide field parallel (horizontal field or HF) and perpendicular (vertical field or VF) to the wave vector $\mathbf{q}$ are $R_{H}$ and $R_{V}$, respectively, the observed neutron cross sections for a paramagnetic system are given by [39, 40]:

$$
\begin{aligned}
\mathrm{HF}: \sigma_{o b}^{+-}= & \frac{R_{H}}{R_{H}+1}\left(M+\frac{2}{3} N S I+B G\right)+ \\
& \frac{1}{R_{H}+1}\left(N+\frac{1}{3} N S I+B G\right) \\
\sigma_{o b}^{++}= & \frac{R_{H}}{R_{H}+1}\left(N+\frac{1}{3} N S I+B G\right)+ \\
& \frac{1}{R_{H}+1}\left(M+\frac{2}{3} N S I+B G\right) \\
\mathrm{VF}: \sigma_{o b}^{+-}= & \frac{R_{V}}{R_{V}+1}\left(\frac{1}{2} M+\frac{2}{3} N S I+B G\right)+ \\
& \frac{1}{R_{V}+1}\left(N+\frac{1}{2} M+\frac{1}{3} N S I+B G\right) \\
\sigma_{o b}^{++}= & \frac{R_{V}}{R_{V}+1}\left(\frac{1}{2} M+N+\frac{1}{3} N S I+B G\right)+ \\
& \frac{1}{R_{V}+1}\left(\frac{1}{2} M+\frac{2}{3} N S I+B G\right)
\end{aligned}
$$

where $M$ is the magnetic, $N$ the nuclear, $N S I$ the nuclear spin incoherent neutron cross section, and $B G$ is the background. Inspection of Eq. [2.2] reveals that the most effective way to detect a magnetic signal is to measure SF scattering with HF. Although such method has been successfully used to identify the magnetic origin of resonance peaks in $\mathrm{YBa}_{2} \mathrm{Cu}_{3} \mathrm{O}_{6+x}$ 14, 18, 19, 20, the advantage of the technique comes at a considerable cost in intensity which makes it impractical for observing small magnetic signals.

As a consequence, most of our experiments were performed with unpolarized neutrons with $\mathrm{Be}$ (002) or PG (002) as monochromators as specified in the figure captions. In these experiments, we also used PG(002) as analyzers. Fast neutrons were removed from the incident beam by an in-pile sapphire filter, and PG filters were used to remove high-order neutron contaminations where appropriate. The horizontal collimations were controlled with Soller slits and are specified in the figures containing the experimental data. To separate the magnetic from phonon scattering, we utilize the differences in their temperature and wave vector dependence of the cross sections. While the phonon scattering gains intensity on warming due to the thermal population factor, the magnetic signal usually becomes weaker because it spreads throughout the energy and momentum space at high temperatures. In addition, magnetic intensity should decrease at large wave vectors due to the reduction in the magnetic form factor while phonon and multiphonon scattering are expected to increase with increasing wave vector. Therefore, in an unpolarized neutron scattering measurement the net intensity gain above the multi-phonon background on cooling at appropriate wave 
vectors is likely to be magnetic in origin. Previous experiments have utilized this method to discover incommensurate spin fluctuations in $\mathrm{YBa}_{2} \mathrm{Cu}_{3} \mathrm{O}_{6.6}$ [22].

Figure 1 depicts the reciprocal lattice of the $\mathrm{YBa}_{2} \mathrm{Cu}_{3} \mathrm{O}_{6+x} \mathbf{a}^{*}(=2 \pi / a), \mathbf{b}^{*}(=2 \pi / b)$ directions shown in square lattice notation. Our crystals are all highly twined so we cannot distinguish $\mathbf{a}^{*}$ from $\mathbf{b}^{*}$. Because the $\mathrm{CuO}_{2}$ planes in $\mathrm{YBa}_{2} \mathrm{Cu}_{3} \mathrm{O}_{6+x}$ actually appear in coupled bilayers that are separated by a much larger distance between the bilayer, spin fluctuations that are in-phase (acoustic or $\chi_{a c}^{\prime \prime}$ ) or out-of-phase (optical or $\chi_{o p}^{\prime \prime}$ ) with respect to the neighboring plane will have different spectra 12, 13, 14. It is therefore convenient to separate the magnetic response of $\mathrm{YBa}_{2} \mathrm{Cu}_{3} \mathrm{O}_{6+x}$ into two parts $\chi^{\prime \prime}\left(q_{x}, q_{y}, q_{z}, \omega\right)=\chi_{a c}^{\prime \prime}\left(q_{x}, q_{y}, \omega\right) \sin ^{2}\left(q_{z} d / 2\right)+$ $\chi_{o p}^{\prime \prime}\left(q_{x}, q_{y}, \omega\right) \cos ^{2}\left(q_{z} d / 2\right)$, where $d(3.342 \AA)$ is the spacing between the nearest-neighbor $\mathrm{CuO}_{2}$ planes along $c$. For the AF insulating parent compound $\mathrm{YBa}_{2} \mathrm{Cu}_{3} \mathrm{O}_{6}, \chi_{a c}^{\prime \prime}$ and $\chi_{o p}^{\prime \prime}$ correspond to the acoustic and optical spin-wave excitations, respectively 41, 42]. Because the interesting features such as the resonance and incommensurate fluctuations $12,13,14,15,16,17,18,19,20,21,22,23$, 24, 25, 26, 27 are only observed in the acoustic channel of spin susceptibility $\chi_{a c}^{\prime \prime}\left(q_{x}, q_{y}, \omega\right)$ and the optical fluctuations occur at higher energies than the acoustic excitations, we focused on the temperature, frequency, and composition dependence of spin excitations in the acoustic mode which has a modulation $\sin ^{2}\left(q_{z} d / 2\right)$ along the $c$-axis.

For this purpose, we used several crystal orientations and scattering geometries. Previous work by different groups $\sqrt{12}, 13,14,15,16,17,18,19,20,21,22,23,24$, 25, 26, 27. were focused on the behavior of magnetic excitations for $\mathbf{q}$ in the $(h, h, l)$ and/or $(h, 3 h, l)$ zone. In view of the fact that the low frequency spin fluctuations in $\mathrm{YBa}_{2} \mathrm{Cu}_{3} \mathrm{O}_{6+x}$ peak at a quartet of incommensurate positions $\delta \mathrm{rlu}$ away from $(1 / 2,1 / 2),(\pi, \pi)$, as depicted in Fig. 1 24, we scanned along the arrow direction in Fig. 1 from $(0,1 / 2)$ to $(1,1 / 2)$ with fixed $l$. To perform these scans, we first aligned the crystal with its $[1,0,0]$ and $[0,0,1]$ axes along the rotational axes of the lower ( $\alpha$-axis) and upper ( $\chi$-axis) arcs of the spectrometer goniometer, respectively. The crystal was then rotated $\alpha=\arctan (c / 2 a l)$ degrees such that after the rotation the $[1 / 2,0,0]$ and $[0,1 / 2, l]$ axes were in the horizontal scattering plane. To access the maxima of acoustic spin fluctuations, we chose $l \approx 2$ or $5 \mathrm{rlu}$ so the $\omega$ rotation angle would be approximately 37 or 17 degrees, respectively. One advantage of performing scans in this manner is that the scan direction is along the direction of incommensurate peaks and the narrow part of the resolution ellipse. As shown in Fig. 1, the narrow part of the spectrometer resolution matches the separation of the incommensurate peaks. Therefore, this is a favorable geometry to study the evolution of the incommensurate magnetic excitations. In addition to the new experimental geometry, we also used the conventional $(h, 3 h, l)$ zone as specified in the figure captions.

\section{RESULTS AND DISCUSSION}

\section{A. Wave vector and energy dependence of the acoustic spin fluctuations}

We begin this section by noting that inelastic neutron scattering experiments over the last decade [12, 13, 14, 15, 16, 17, 18, 19, 20, 21, 22, 23, 24, 25, 26, 27 have provided valuable information about the evolution of the spin dynamics in $\mathrm{YBa}_{2} \mathrm{Cu}_{3} \mathrm{O}_{6+x}$. The key features of the spin excitations spectra include a gap in the superconducting state, a pseudogap in the normal state, the resonance peak, and the incommensurate fluctuations below the resonance. The observation of remarkable similarities in spin incommensurate structure for the bilayer $\mathrm{YBa}_{2} \mathrm{Cu}_{3} \mathrm{O}_{6.6} 24$ and the single-layer $\mathrm{La}_{2-x} \mathrm{Sr}_{x} \mathrm{CuO}_{4}$ of the same hole doping density [8] suggests that incommensurate magnetic fluctuations may be a universal feature of the cuprate superconductors. For the $\mathrm{La}_{2-x} \mathrm{Sr}_{x} \mathrm{CuO}_{4}$ system, Yamada and coworkers established the Sr-doping dependence of the incommensurability $\delta$ of the spin fluctuations and found a remarkable linear relationship between $T_{c}$ and $\delta\left[8\right.$. In the case of $\mathrm{YBa}_{2} \mathrm{Cu}_{3} \mathrm{O}_{6+x}$, Balatsky and Bourges 43. suggested that a similar linear relationship exists between $T_{c}$ and the width in momentum space $(q)$ of the "normal" spin fluctuations, i.e., fluctuations at frequencies away from the resonance. The authors argued that spin excitations of $\mathrm{YBa}_{2} \mathrm{Cu}_{3} \mathrm{O}_{6+x}$ have two components and the low-energy incommensurate fluctuations are different than the commensurate resonance. The conclusions of [43] were reached based mostly on data where explicit incommensurability in the low-frequency spin fluctuations was not observed. Therefore, it is still not clear whether the linear relationship between $T_{c}$ and $\delta$ is a universal feature of the cuprates. Below, we present our systematic investigation of spin excitations in $\mathrm{YBa}_{2} \mathrm{Cu}_{3} \mathrm{O}_{6+x}$.

\section{1. $\mathrm{YBa}_{2} \mathrm{Cu}_{3} \mathrm{O}_{6.45}$}

As stressed in Sec. IIB, it is not a trivial matter to separate the magnetic scattering from phonon and other spurious processes in metallic crystals of $\mathrm{YBa}_{2} \mathrm{Cu}_{3} \mathrm{O}_{6+x}$. Although the use of polarized neutrons can provide an unambiguous measure of the magnetic and phonon scattering [39], the technique usually suffers from the limited flux available with polarized neutrons. Nevertheless, polarization analysis has distinctive advantages in separating the magnetic signal from phonon and other spurious processes. As an example, we show in Figure 3 the polarization analysis of the spin excitations for $\mathrm{YBa}_{2} \mathrm{Cu}_{3} \mathrm{O}_{6.45}$ at temperatures above and below $T_{c}$.

Because magnetic fluctuations in $\mathrm{YBa}_{2} \mathrm{Cu}_{3} \mathrm{O}_{6+x}$ are centered around $(\pi, \pi)$ with a sinusoidal modulation along the $(0,0, l)$ direction, we oriented the crystal in the $(h, 3 h, l)$ zone and searched for the magnetic signal with scans along $(h, 3 h,-1.7)$ which corresponds to the 


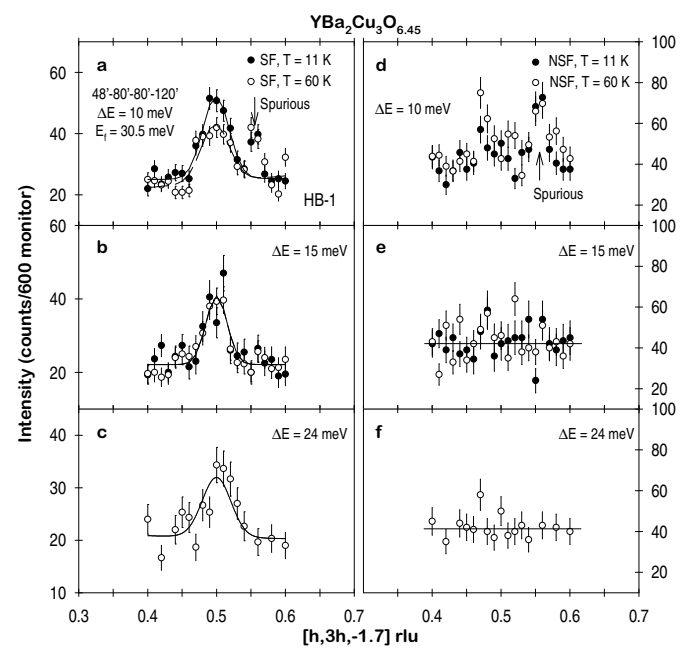

Figure 3
Dai et al.

FIG. 3: The spin-flip (SF) scattering of $\mathrm{YBa}_{2} \mathrm{Cu}_{3} \mathrm{O}_{6.45}$ in the polarized scans along $(H, 3 H,-1.7)$ direction with $\hbar \omega=10$ $\mathrm{meV}$ (a), $15 \mathrm{meV}$ (b), and $24 \mathrm{meV}$ (c) at $11 \mathrm{~K}$ and $60 \mathrm{~K}$. The nonspin-flip (NSF) scattering of the same scans at $\hbar \omega=10$ $\mathrm{meV}, 15 \mathrm{meV}$, and $24 \mathrm{meV}$ are shown in (d), (e), and (f), respectively. The solid and dashed lines in (a-c) are Gaussian fits to the data on linear backgrounds. The solid lines in (e) and (f) are guides to the eye.

maximum intensity of the acoustic modulation. Figures $3(\mathrm{a}-\mathrm{c})$ show the raw data of SF scattering for $\hbar \omega=10$, 15 , and $24 \mathrm{meV}$ at 60 and $11 \mathrm{~K}$. The NSF scattering of the same scans are shown in Figs. 3(d-f). While SF scattering clearly peaks around $(\pi, \pi)$, there are no similar features in the NSF scattering at the same position. Therefore, it is clear that the scattering around $(\pi, \pi)$ in the SF scattering are magnetic in origin. To quantitatively parameterize the SF scattering data, we used a Gaussian line shape on a linear background to fit the data as shown in solid lines in Figs. 3(a-c) [12, 13, 14, 15, 16, 17, 18, 19, 20, 21. The scattering function $S(\mathbf{q}, \omega)$ for such line shape is related to the inplane dynamical spin susceptibility $\chi_{a c}^{\prime \prime}\left(q_{x}, q_{y}, \omega\right)$ by

$$
\begin{aligned}
S(\mathbf{q}, \omega) & =I(\omega) \sin ^{2}\left(q_{z} d / 2\right) \chi_{a c}^{\prime \prime}\left(q_{x}, q_{y}, \omega\right) \\
& =I(\omega) \sin ^{2}\left(q_{z} d / 2\right) e^{\left[-\Delta q^{2} / 2 \sigma^{2}\right]}
\end{aligned}
$$

where $\Delta \mathbf{q}=2 \pi(h-1 / 2,3 h-3 / 2,0) / a, I(\omega)$ is the peak susceptibility and $\sigma$ is related to the half width at half maximum (HWHM) of the excitations via $\mathrm{HWHM}=$ $\sigma \sqrt{2 \ln 2}$.

Although polarization analysis is excellent in identify-

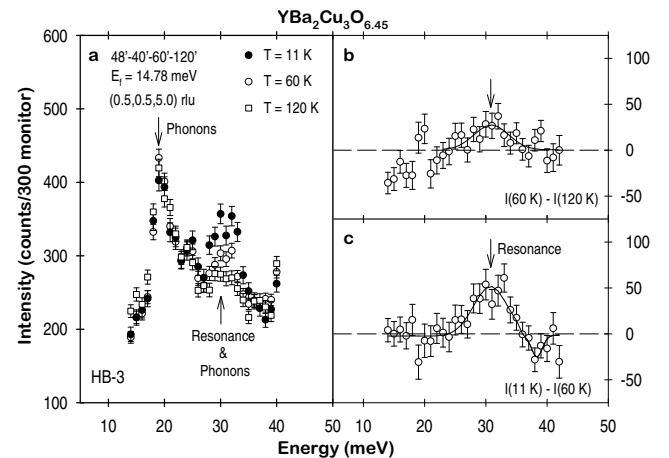

Figure 4
Dai et al.

FIG. 4: The unpolarized constant-q scans at $(0.5,0.5,5)$ rlu for $\mathrm{YBa}_{2} \mathrm{Cu}_{3} \mathrm{O}_{6.45}$. Data were taken with a $\mathrm{PG}(002)$ monochromator and PG analyzer with $40^{\prime}-40^{\prime}-60^{\prime}-120^{\prime}$ collimation and $E_{f}=14.78 \mathrm{meV}$. (a) Raw scattering at three different temperatures with phonons marked by arrows. (b) The temperature difference between $60 \mathrm{~K}\left(T_{c}+12 \mathrm{~K}\right)$ and 120 $\mathrm{K}$. The positive scattering around $30 \mathrm{meV}$ is consistent with the precursor of the resonance in the normal state. (c) The temperature difference between $11 \mathrm{~K}\left(T_{c}-37 \mathrm{~K}\right)$ and $60 \mathrm{~K}$. The solid lines are Gaussian fits to the data. The resonance intensity gain at $11 \mathrm{~K}$ is partially compensated by the loss of the spectral weight at energies above it.

ing the magnetic nature of Gaussian peaks around $(\pi, \pi)$, the limited flux available with the technique means that detailed wave vector and energy dependence of the scattering need to be obtained with unpolarized neutrons. For this purpose, we realigned the sample in the $[1 / 2,0,0]$ and $[0,1 / 2, l]$ zone. Figure 4 shows the constant- $q$ scans at various temperatures at $(\pi, \pi)$. The spectra consist of two major peaks at $\sim 19$ and $31 \mathrm{meV}$. While the intensity of the $\sim 19 \mathrm{meV}$ mode follows the Bose population factor $[n(\omega)+1]$ consistent with phonons, the scattering at $\sim 30 \mathrm{meV}$ decreases with warming which is indicative of its magnetic origin. As we shall demonstrate below, phonons are also present at $\sim 31 \mathrm{meV}$. However, phonon scattering in this energy range is expected to change negligibly with temperature for $T \leq 120 \mathrm{~K}$. Therefore, the difference spectra in Fig. 4 can be regarded as changes in the dynamical susceptibility. Clearly, there are spectral weight enhancements around $30 \mathrm{meV}$ with decreasing temperature above and below $T_{c}$. If we define the suscep- 


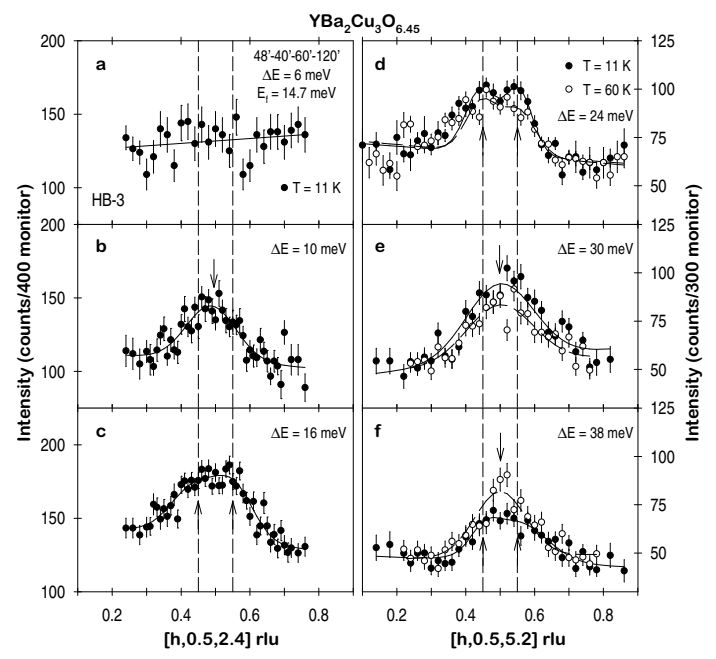

Figure 5
Dai et al.

FIG. 5: Constant-energy scans along the $[h, 0.5,2.4]$ (a-c) and $[h, 0.5,5.2]$ (d-f) directions. The energy transfers are $\hbar \omega=6 \mathrm{meV}$ (a), $10 \mathrm{meV}$ (b), $16 \mathrm{meV}$ (c), $24 \mathrm{meV}$ (d), 30 $\mathrm{meV}$ (e), and $38 \mathrm{meV}$ (f). Incommensurate spin fluctuations are clearly observed at $\hbar \omega=24 \mathrm{meV}$ both above and below $T_{c}$. In the superconducting state, the flattish top profiles are observed at energies above and below the resonance energy. Solid lines are Gaussian fits to the data in (b-f).

tibility gain on cooling above $T_{c}$ at the resonance energy as the precursor of the resonance, we find that the spectral weight of the resonance increases slightly from the normal to the superconducting state [Fig. 4(b) and (c)] and is only partially compensated by the loss at energies above it [Fig. 4(c)].

Figure 5 shows the momentum dependence of the response at energies above and below the magnetic resonance. At $\hbar \omega=6 \mathrm{meV}$, the scattering [Fig. 5(a)] is featureless around $(\pi, \pi)$, suggesting the presence of a spin gap in the superconducting state [44]. On increasing the energy to $10 \mathrm{meV}$, the scattering shows a broad peak that can be well described by a single Gaussian centered at $(\pi, \pi)$. At $\hbar \omega=16 \mathrm{meV}$, the profile around $(\pi, \pi)$ develops a flattish top similar to previous observations [13, 15. Although detailed analysis suggests that the profile is better described by a pair of peaks rather than a single Gaussian, well defined incommensurate peaks at $\delta=0.054 \pm 0.004 \mathrm{rlu}$ from $(\pi, \pi)$ are only observed at $\hbar \omega=24 \mathrm{meV}$ [Fig. 5(d)]. At the resonance energy of $\sim 30 \mathrm{meV}$, commensurate profiles are observed above and below $T_{c}$ [Fig. 5(e)]. For an energy above the resonance (at $38 \mathrm{meV}$ ), the peak appears to be commensurate in the normal state, but changes to a flat top (incommensurate) profile in the low-temperature superconducting state [Fig. 5(f)].

\section{2. $\mathrm{YBa}_{2} \mathrm{Cu}_{3} \mathrm{O}_{6.5}, \mathrm{YBa}_{2} \mathrm{Cu}_{3} \mathrm{O}_{6.6}$, and $\mathrm{YBa}_{2} \mathrm{Cu}_{3} \mathrm{O}_{6.7}$}

In this section, we discuss results on three samples with superconducting transition temperatures of $52 \mathrm{~K}, 62.7$ $\mathrm{K}$, and $74 \mathrm{~K}$. The fractional hole concentration per $\mathrm{Cu}$ atom in the $\mathrm{CuO}_{2}$ sheet, $p$, for $\mathrm{YBa}_{2} \mathrm{Cu}_{3} \mathrm{O}_{6+x}$ around the $60-\mathrm{K}$ plateau phase is close to $\sim 0.1$ [45]. For the single-layer superconducting $\mathrm{La}_{1.9} \mathrm{Sr}_{0.1} \mathrm{CuO}_{4}$ which also has $p=0.1$, the incommensurability of the spin fluctuations is at $\delta=0.1$ [ 8$]$. The discovery of the same incommensurate structure and $\delta(\approx 0.1)$ for the equivalent hole concentration $(p \approx 0.1)$ of the bilayer $\mathrm{YBa}_{2} \mathrm{Cu}_{3} \mathrm{O}_{6.6} 24$ has generated considerable interests because such observation suggests that incommensurate spin fluctuations may be the common feature of these two most studied families of cuprates.

For $\mathrm{La}_{2-x} \mathrm{Sr}_{x} \mathrm{CuO}_{4}$, it is generally believed that the presence of dynamic stripes is the microscopic origin of the observed incommensurate spin fluctuations [46. Thus, if the incommensurate spin fluctuations in $\mathrm{YBa}_{2} \mathrm{Cu}_{3} \mathrm{O}_{6+x}$ have similar behavior as $\mathrm{La}_{2-x} \mathrm{Sr}_{x} \mathrm{CuO}_{4}$ at all doping concentrations, it is likely that dynamic stripes should also be present in the bilayer cuprates. Although the explicit incommensurate structure [24 and the one-dimensional nature of the incommensurate spin fluctuations in $\mathrm{YBa}_{2} \mathrm{Cu}_{3} \mathrm{O}_{6.6}$ 47 favor the dynamic striped-phase interpretation, the detailed energy and doping dependence of the incommensurate spin fluctuations is still lacking. In the discussions below, we attempt to remedy this situation.

We first describe measurements on $\mathrm{YBa}_{2} \mathrm{Cu}_{3} \mathrm{O}_{6.5}$. Although the rocking curves of all other crystals of $\mathrm{YBa}_{2} \mathrm{Cu}_{3} \mathrm{O}_{6+x}$ show single Gaussian peaks with mosaic spreads of $\sim 1-2.5$ degrees, the rocking curve around $(0,0,6)$ Bragg reflection for $\mathrm{YBa}_{2} \mathrm{Cu}_{3} \mathrm{O}_{6.5}$ displays two distinct peaks separated by $\sim 3$ degrees. This means that the $\mathrm{YBa}_{2} \mathrm{Cu}_{3} \mathrm{O}_{6.5}$ sample is composed of two major grains $\sim 3$ degrees apart. Figure 6 summarizes constant- $q$ scans at various temperatures at $(\pi, \pi)$ for $\mathrm{YBa}_{2} \mathrm{Cu}_{3} \mathrm{O}_{6.5}$. While the $\sim 20 \mathrm{meV}$ phonons change negligibly from those for $\mathrm{YBa}_{2} \mathrm{Cu}_{3} \mathrm{O}_{6.45}$ (Fig. 4), there are two features in the raw spectra of Fig. 6(a) worth noting. First, the resonance energy shifted from $\sim 30.5 \mathrm{meV}$ for $\mathrm{YBa}_{2} \mathrm{Cu}_{3} \mathrm{O}_{6.45}$ to $\sim 32 \mathrm{meV}$ for $\mathrm{YBa}_{2} \mathrm{Cu}_{3} \mathrm{O}_{6.5}$. In addition, the spectral weight of the resonance relative to that of the $20 \mathrm{meV}$ phonons is clearly enhanced in $\mathrm{YBa}_{2} \mathrm{Cu}_{3} \mathrm{O}_{6.5}$. This observation indicates that the intensity gain of the resonance in this material below $T_{c}$ is considerably bigger than that for $\mathrm{YBa}_{2} \mathrm{Cu}_{3} \mathrm{O}_{6.45}$. Similar to $\mathrm{YBa}_{2} \mathrm{Cu}_{3} \mathrm{O}_{6.45}$, there are magnetic spectral weight enhancements around the resonance energy above and below $T_{c}$. High intensity, low-resolution measurements in the $[h, 3 h, l]$ zone confirm this conclusion (Fig. 7 ). 


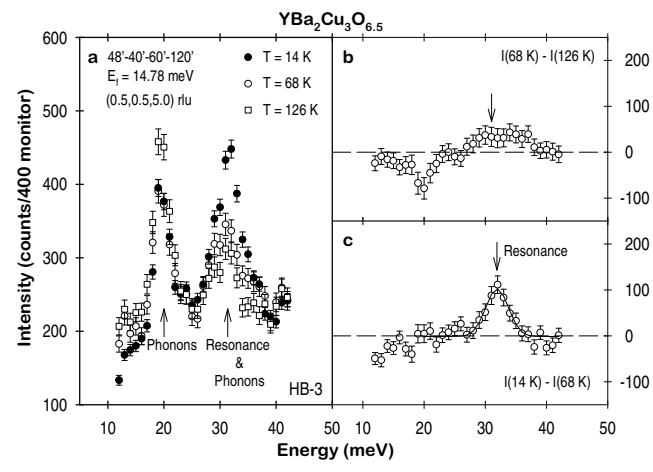

Figure 6
Dai et al.

FIG. 6: Constant-q scans at $(0.5,0.5,5)$ rlu for $\mathrm{YBa}_{2} \mathrm{Cu}_{3} \mathrm{O}_{6.5}$. (a) Raw scattering at three different temperatures with phonons and resonance marked by arrows. (b) The temperature difference between $68 \mathrm{~K}\left(T_{c}+16 \mathrm{~K}\right)$ and $126 \mathrm{~K}$. The positive scattering around $32 \mathrm{meV}$ is consistent with the precursor of the resonance in the normal state. (c) The temperature difference between $14 \mathrm{~K}\left(T_{c}-38 \mathrm{~K}\right)$ and $68 \mathrm{~K}$. The solid lines are Gaussian fits to the data.

To determine the magnitude of the spin gap and search for incommensurate spin fluctuations, we performed constant-energy scans at various energies along the $[h, 0.5,5]$ direction above and below $T_{c}$ as shown in Fig. 8. At 10 and $16 \mathrm{meV}$, the scattering shows weak features around $(\pi, \pi)$. This is different from $\mathrm{YBa}_{2} \mathrm{Cu}_{3} \mathrm{O}_{6.45}$ where spin fluctuations centered around $(\pi, \pi)$ are found at energies above $10 \mathrm{meV}$ (Figs. 3 and 5). At $24 \mathrm{meV}$ [Fig. 8(c)], the scattering displays a flattish top in both the normal and superconducting states [48]. At energies near [27 meV, Fig. 8(d)] and at the resonance [31.5 meV, Fig. 8(e)], the scattering narrows in width and increases in intensity. For an energy above the resonance, flattish top profiles indicative of incommensurate fluctuations are observed at $40 \mathrm{meV}$ as shown in Fig. 8(f), which is similar to Fig. 5(f) of $\mathrm{YBa}_{2} \mathrm{Cu}_{3} \mathrm{O}_{6.45}$.

Next we describe measurements on $\mathrm{YBa}_{2} \mathrm{Cu}_{3} \mathrm{O}_{6.6}$ where the incommensurate spin fluctuations at $24 \mathrm{meV}$ and the resonance at $34 \mathrm{meV}$ have been extensively studied [19, 22, 23, 24, 27]. For completeness, we plot in Fig. 9 the difference spectra of constant- $q$ scans showing the presence of the $34 \mathrm{meV}$ resonance above and below $T_{c}$. Detailed temperature evolution of the resonance and the

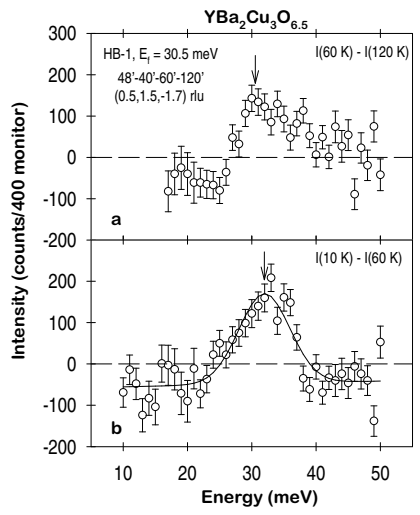

Figure 7
Dai et al.

FIG. 7: Constant-q scans at $(0.5,1.5,-1.7)$ rlu above and below $T_{c}$ for $\mathrm{YBa}_{2} \mathrm{Cu}_{3} \mathrm{O}_{6.5}$ with the crystal in the $[h, 3 h, l]$ zone. (a) Difference scattering between $60 \mathrm{~K}\left(T_{c}+8 \mathrm{~K}\right)$ and $120 \mathrm{~K}$. (b) Difference scattering between $10 \mathrm{~K}\left(T_{c}-42 \mathrm{~K}\right)$ and $60 \mathrm{~K}$. The solid lines are Gaussian fits to the data.

magnitude of the spin gap has already been reported [27. To establish the energy dependence of the incommensurate spin fluctuations, we carried out high-resolution measurements using $\mathrm{Be}(002)$ as the monochromator and fixed the final neutron energy at $14.78 \mathrm{meV}$. Figure 10 shows a series of constant-energy scans at energies below and above the $34 \mathrm{meV}$ resonance. Consistent with previous results [22, 23, 24, constant-energy scans at $24 \mathrm{meV}$ [Fig. 10(a)] show a pair of well-defined peaks separated by $2 \delta=0.20 \pm 0.01 \mathrm{rlu}$ in the low-temperature superconducting state and a flattish top profile just above $T_{c}$. Although the same scans at $27 \mathrm{meV}$ and $30 \mathrm{meV}$ also exhibit the same double peak structure below $T_{c}$ [Figs. 10 (b) and (c)], the separation between the peaks or the incommensurabilities clearly decrease with increasing energy. In addition, the normal state flat top profile at $24 \mathrm{meV}$ is replaced by a single Gaussian peak centered around $(\pi, \pi)$ at $30 \mathrm{meV}$. As energy increases, the scattering profile continues to sharpen, with the narrowest width and highest intensity profile occuring at the resonance energy [Fig. 10(e)]. When moving to energies above the resonance [Figs. 10(f-h)], the profiles increase in width and decrease in intensity. At $41 \mathrm{meV}$, the scattering becomes incommensurate in both the normal and superconducting states [Fig. 10(h)].

Finally, we discuss measurements for $\mathrm{YBa}_{2} \mathrm{Cu}_{3} \mathrm{O}_{6.7}$. 


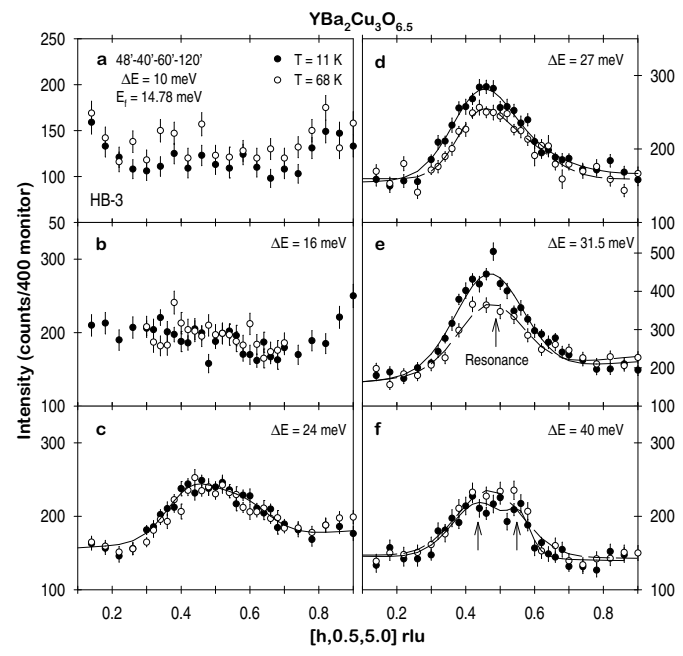

Figure 8
Dai et al.

FIG. 8: Constant-energy scans along the $[h, 0.5,5.0]$ direction for $\mathrm{YBa}_{2} \mathrm{Cu}_{3} \mathrm{O}_{6.5}$. The energy transfers are $\hbar \omega=10 \mathrm{meV}$ (a), $16 \mathrm{meV}$ (b), $24 \mathrm{meV}$ (c), $27 \mathrm{meV}$ (d), $31.5 \mathrm{meV}$ (e), and 40 $\mathrm{meV}(\mathrm{f})$. The flattish top profiles indicative of the incommensurate spin fluctuations are observed at $\hbar \omega=24 \mathrm{meV}$ both above and below $T_{c}$. In the superconducting state, flattish top profiles are also observed above the resonance $\hbar \omega=40 \mathrm{meV}$. The solid lines are Gaussian fits to the data, and the arrows in (c) and (f) mark the fitted incommensurate positions.

Figure 11 shows the result of constant- $q$ scans above and below $T_{c}$. As the energy of the resonance moves up to $37 \mathrm{meV}$, the phonon scattering at $31 \mathrm{meV}$ becomes more prominent. Constant-energy scans at several energies below the resonance are shown in Fig. 12. In contrast to the well-defined double peaks for $\mathrm{YBa}_{2} \mathrm{Cu}_{3} \mathrm{O}_{6.6}$ [Fig. 10(a)], the scattering at $24 \mathrm{meV}$ and $27 \mathrm{meV}$ [Figs. 12(a) and (b)] shows a very weak structure above and below $T_{c}$. For $\mathrm{YBa}_{2} \mathrm{Cu}_{3} \mathrm{O}_{6.6}$, the susceptibility increases on cooling from the normal to the superconducting state at the incommensurate positions, accompanied by a suppression of fluctuations at the commensurate point [22]. In an attempt to determine whether the same is true for $\mathrm{YBa}_{2} \mathrm{Cu}_{3} \mathrm{O}_{6.7}$, we plot the difference spectra between 11 $\mathrm{K}$ and $80 \mathrm{~K}$. As emphasized in an earlier study [22], these difference spectra can be simply regarded as changes in the dynamical susceptibility, i.e., $\chi^{\prime \prime}(11 K)-\chi^{\prime \prime}(80 K)$. Figures $12(\mathrm{~d})$ and (e) show the outcome of this subtraction for $24 \mathrm{meV}$ and $27 \mathrm{meV}$, respectively. For $\hbar \omega=24$ $\mathrm{meV}$, the difference spectrum shows negative scattering centered around $(\pi, \pi)$ with no evidence of susceptibility gain at the expected incommensurate positions. This

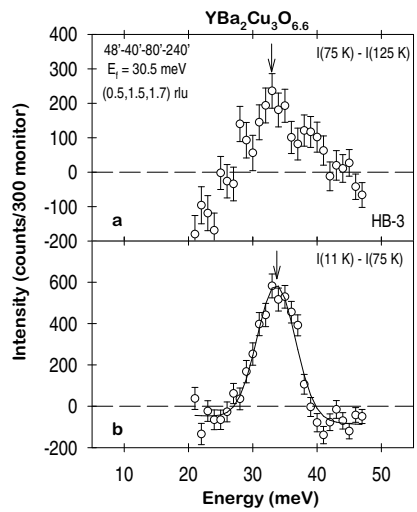

Figure 9
Dai et al.

FIG. 9: Constant-q scans at $(0.5,1.5,1.7)$ rlu above and below $T_{c}$ for $\mathrm{YBa}_{2} \mathrm{Cu}_{3} \mathrm{O}_{6.6}$ with the crystal in the $[h, 3 h, l]$ zone. (a) The temperature difference between $75 \mathrm{~K}\left(T_{c}+12 \mathrm{~K}\right)$ and 125 $\mathrm{K}$. (b) The temperature difference between $11 \mathrm{~K}\left(T_{c}-52 \mathrm{~K}\right)$ and $75 \mathrm{~K}$. The solid line is a Gaussian fit to the data.

is consistent with the opening of a $24 \mathrm{meV}$ gap in the spin fluctuations spectra below $T_{c}$. At $\hbar \omega=27 \mathrm{meV}$, both the raw data [Fig. 12(b)] and the difference spectrum show evidence of incommensurate spin fluctuations at $\delta \approx 0.1 \mathrm{rlu}$, consistent with previous measurements using an integrated technique that gives a incommensurability of $\delta=0.11 \pm 0.01$ [23. However, the clarity of incommensurate spin fluctuations is weak as compared to that for $\mathrm{YBa}_{2} \mathrm{Cu}_{3} \mathrm{O}_{6.6}$. Experiments on a different crystal of $\mathrm{YBa}_{2} \mathrm{Cu}_{3} \mathrm{O}_{6.7}$ by Arai et al. [25] show clear incommensurate spin fluctuations at $\delta=0.11 \pm 0.01 \mathrm{rlu}$. At energies close to the resonance $(\hbar \omega=34 \mathrm{meV}$ and 37 $\mathrm{meV}$ ), the scattering [Figs. 12(c) and (f)] shows conventional Gaussian profiles.

\section{3. $\mathrm{YBa}_{2} \mathrm{Cu}_{3} \mathrm{O}_{6.8}$}

Much of the discussion in this section will be focused on our analysis of separating phonon and spurious events from magnetic scattering for energies below the resonance. While the magnetic signal usually spreads throughout the energy and momentum space at high temperatures and becomes unobservable at any particular $\hbar \omega-Q$ point, the intensity of phonons should follow the Bose statistics with increasing temperature. Therefore, 


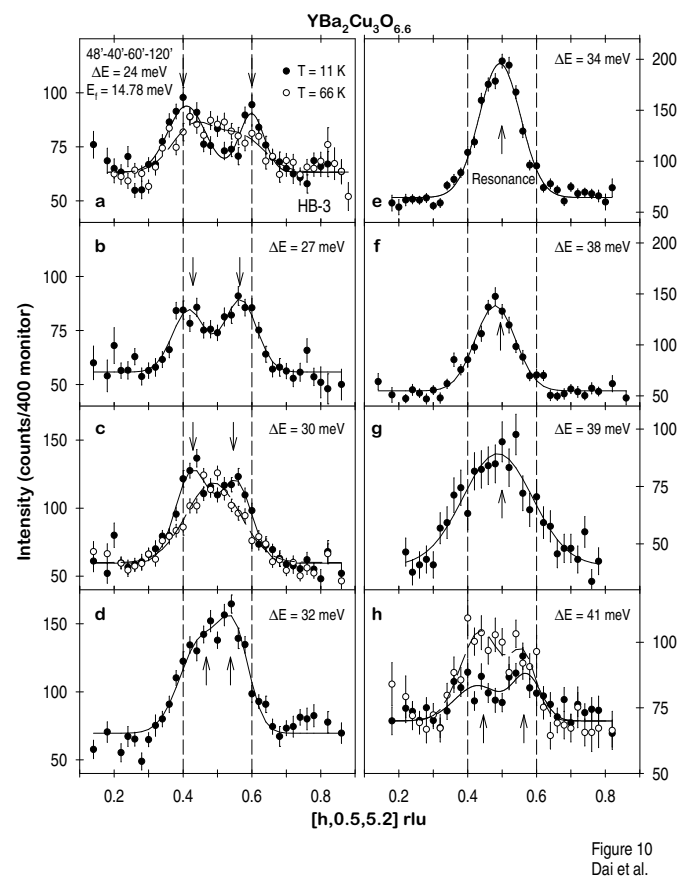

FIG. 10: Constant-energy scans along the $[h, 0.5,5.2]$ direction for $\mathrm{YBa}_{2} \mathrm{Cu}_{3} \mathrm{O}_{6.6}$. The data were taken with a $\mathrm{Be}(002)$ monochromator and a PG analyzer with a neutron final energy fixed at $E_{f}=14.78 \mathrm{meV}$. The energy transfers are $\hbar \omega=24 \mathrm{meV}$ (a), $27 \mathrm{meV}$ (b), $30 \mathrm{meV}$ (c), $32 \mathrm{meV}$ (d), 34 $\mathrm{meV}$ (e), $38 \mathrm{meV}$ (f), $39 \mathrm{meV}$ (g), and $41 \mathrm{meV}(\mathrm{h})$. The flattish top profiles indicative of the incommensurate spin fluctuations are clearly observed in the normal state at $\hbar \omega=24$ $\mathrm{meV}$ and $41 \mathrm{meV}$. However, at $\hbar \omega=30 \mathrm{meV}$, the scattering is commensurate in the normal state. In the superconducting state, the incommensurability of spin fluctuations decreases with increasing energy as the resonance is approached and splits up again at energies above the resonance. The solid lines are Gaussian fits to the data.

careful measurements as a function of temperature and wave vector should allow the distinction of magnetic scattering from phonons.

As an example, we show in Fig. 13 raw constant- $Q$ scans at the wave vector $\mathbf{q}=(0.5,0.5,5)$ rlu at various temperatures. The comparison of the results for temperatures above and below $T_{c}$ [Fig. 13(b)] indicates a clear enhancement of the scattering at $\hbar \omega \approx 40 \mathrm{meV}$, consistent with the presence of the resonance. In addition to the enhancement of the resonance, there are general intensity reductions in the scattering below $\sim 30 \mathrm{meV}$ from the normal to the superconducting state. Such intensity reductions at low energies are likely due to the drop in the single- and multi- phonon scattering although a simultaneous reduction in magnetic signal cannot be ruled out. Nevertheless, the strong enhancement of the scattering at $\hbar \omega=\sim 20, \sim 31$, and $\sim 41 \mathrm{meV}$ at $200 \mathrm{~K}$ [Fig. 13(a)] indicates that the peaks in the constant- $Q$ scans at these

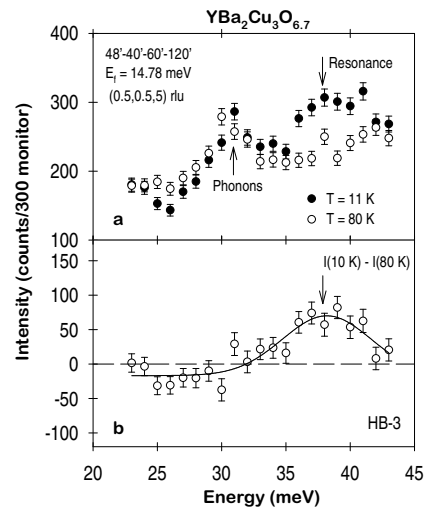

Figure 11
Dai et al.

FIG. 11: Constant-q scans at $(0.5,0.5,5)$ rlu above and below $T_{c}$ for $\mathrm{YBa}_{2} \mathrm{Cu}_{3} \mathrm{O}_{6.7}$. (a) Raw scattering at two different temperatures with phonons and resonance marked by arrows. (b) The temperature difference between $10 \mathrm{~K}\left(T_{c}-64 \mathrm{~K}\right)$ and $80 \mathrm{~K}$. The positive scattering around $37 \mathrm{meV}$ shows the resonance. The solid line is a Gaussian fit to the data.

energies are mostly phonons in origin. To demonstrate the enhancement of the magnetic scattering on cooling above and below $T_{c}$, we show the difference spectra between low and high temperatures in two experimental geometries in Figs. 14 and 15. The data reveal two important features: first, the resonance in this underdoped compounds occurs at $\sim 40 \mathrm{meV}$, almost identical to the optimally and overdoped doped compound [12, 14, 16; second, there is an enhancement of the magnetic signal around the resonance energy above $T_{c}$, consistent with the presence of a precursor for the resonance in the underdoped $\mathrm{YBa}_{2} \mathrm{Cu}_{3} \mathrm{O}_{6+x}$ 27.

To search for incommensurate spin fluctuations in $\mathrm{YBa}_{2} \mathrm{Cu}_{3} \mathrm{O}_{6.8}$, we scanned along the wave vector $[h, 0.5,5]$ direction at energies below and above the resonance. Figure 16 summarizes the low-resolution raw data at temperatures just above and well below $T_{c}$. At $\hbar \omega=16 \mathrm{meV}$ [Fig. 16(a)], the scattering shows a sloped background with minimum intensity at around $(\pi, \pi)$ in both the normal and superconducting states. This suggests the existence of a normal state spin gap of $16 \mathrm{meV}$. On increasing the energy to $\hbar \omega=24 \mathrm{meV}$ [Fig. 16(b)], the intensity of the broad peak around $(\pi, \pi)$ in the normal state is somewhat reduced below $T_{c}$ with no clear evidence of an incommensurate structure. A similar be- 


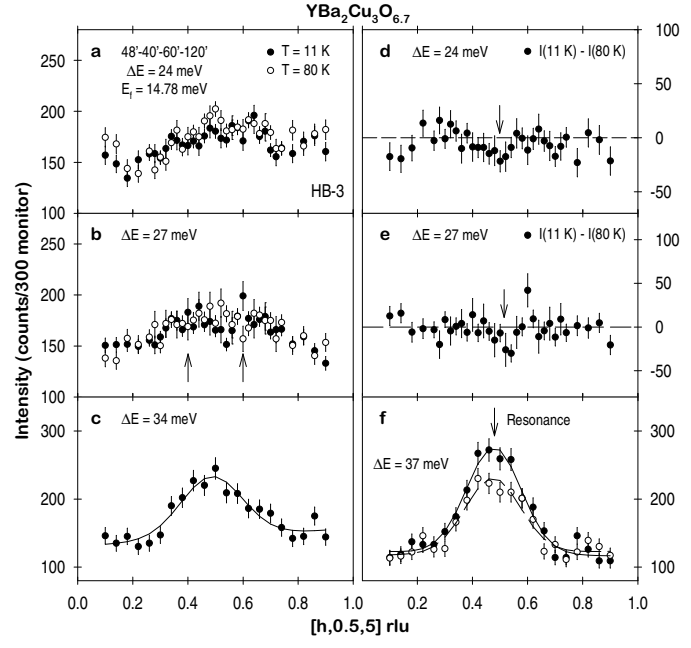

Figure 12
Dai et al.

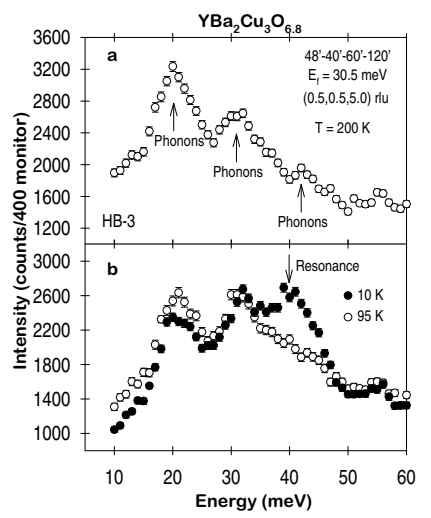

Figure 13
FIG. 12: Constant-energy scans along the $[h, 0.5,5.0]$ direction for $\mathrm{YBa}_{2} \mathrm{Cu}_{3} \mathrm{O}_{6.7}$. The energy transfers are $\hbar \omega=24$ $\mathrm{meV}$ (a), $27 \mathrm{meV}$ (b), $34 \mathrm{meV}$ (c), and $37 \mathrm{meV}$ (f). (d) and (e) show the difference spectra between $11 \mathrm{~K}$ and $80 \mathrm{~K}$. In the superconducting state, weak incommensurate peaks are observed at $\hbar \omega=27 \mathrm{meV}$ (b), and a spin gap opens up at 24 meV (d). The solid lines are Gaussian fits to the data, and the arrows in (b) and (e) indicate the incommensurate positions. The integrated method described in Ref. [23] confirms the present measurements.

havior is also observed at $\hbar \omega=28 \mathrm{meV}$ [Figs. 16(c)]. The scattering profiles between the normal and superconducting states are almost indistinguishable at $\hbar \omega=32$ $\mathrm{meV}$ and $52 \mathrm{meV}$ [Figs. 16(d) and (f)]. Near the resonance energy [Fig. 16(e)], the scattering can be well described by Gaussians on linear backgrounds and shows a clear increase in intensity on entering the superconducting state from the normal state. In addition, the scattering at $200 \mathrm{~K}$ (open squares) shows a broad peak around $(\pi, \pi)$, which indicates weak magnetic scattering at this temperature.

Since the scattering at $200 \mathrm{~K}$ is mostly nonmagnetic for energies below $39 \mathrm{meV}$ [Fig. 16(e)], we systematically subtracted $200 \mathrm{~K}$ data from that obtained at lower temperatures. Figures 17(c-f) show the outcome of such procedure, and the net intensity gain around $(\pi, \pi)$ above the general reduction in the multi-phonon background should represent the enhancement of magnetic scattering at low temperatures. For $\hbar \omega=28 \mathrm{meV}$ [Fig. 17(c)], the broad peak in the normal state vanishes below $T_{c}$, which suggests the enlargement of the normal state spin
FIG. 13: Constant-q scans at $(0.5,0.5,5)$ rlu for $\mathrm{YBa}_{2} \mathrm{Cu}_{3} \mathrm{O}_{6.8}$. (a) Raw scattering at $200 \mathrm{~K}$ with phonons marked by arrows. (b) The same scans at $10 \mathrm{~K}$ and $95 \mathrm{~K}$ with the resonance marked by an arrow.

gap in the superconducting state. On increasing the energy to $\hbar \omega=32 \mathrm{meV}$, the scattering shows little change from the normal state to the superconducting state [Figs. $17(\mathrm{~d})$ ]. In addition, the maximum scattering intensity no longer seems to peak at $(\pi, \pi)$, but shows weak evidence for incommensurate scattering. Unfortunately, the poor resolution of the measurements does not allow a conclusive identification of incommensurate spin fluctuations. At $\hbar \omega=39 \mathrm{meV}$ [Fig. $17(\mathrm{e})]$, the enhancement of resonance in Fig. 16(e) is confirmed. Finally for an energy above the resonance $(\hbar \omega=52 \mathrm{meV})$, the scattering shows no observable change above and below $T_{c}$ [Fig. 17(f)].

Because the intensity of low energy phonons is more sensitive to the increasing temperature and the Bose population factor, the subtraction procedure described above does not work for low energy excitations. However, we can compare the difference spectra at temperatures just above and below $T_{c}$. At $\hbar \omega=16 \mathrm{meV}$ [Fig. 17(a)], the difference spectra between $95 \mathrm{~K}\left(T_{c}+13 \mathrm{~K}\right)$ and 10 $\mathrm{K}\left(<T_{c}\right)$ show no identifiable feature which is consistent with the presence of a normal state spin gap. Similar subtraction at $\hbar \omega=24 \mathrm{meV}$ shows a clear peak around $(\pi, \pi)$ [Fig. 17(b)] which indicates the suppression of $24 \mathrm{meV}$ spin fluctuations in the superconducting state. Therefore, the normal state spin fluctuations in $\mathrm{YBa}_{2} \mathrm{Cu}_{3} \mathrm{O}_{6.8}$ consist of a gap $(\geq 16 \mathrm{meV})$ and broad excitations centered around $(\pi, \pi)$. In the superconducting state, the 


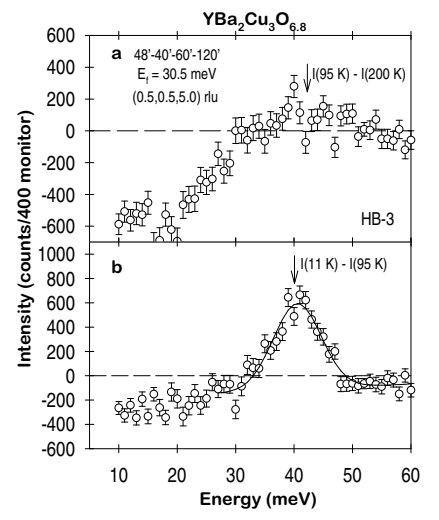

Figure 14
Dai et al.

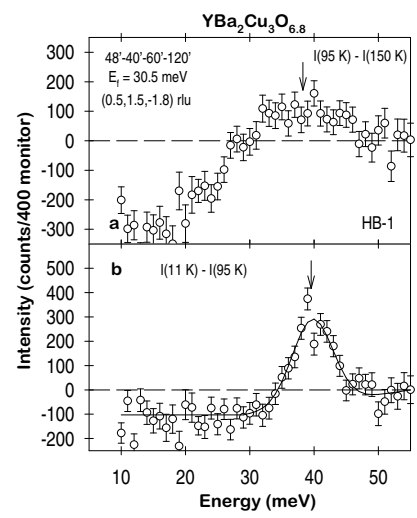

Figure 15
Dai et al.
FIG. 14: Difference spectra in constant-q scans at $(0.5,0.5,5)$ rlu above and below $T_{c}$ for $\mathrm{YBa}_{2} \mathrm{Cu}_{3} \mathrm{O}_{6.8}$. (a) The temperature difference between $95 \mathrm{~K}\left(T_{c}+13 \mathrm{~K}\right)$ and $200 \mathrm{~K}$. The positive scattering around $40 \mathrm{meV}$ is consistent with the precursor of the resonance in the normal state. (b) The temperature difference between $11 \mathrm{~K}\left(T_{c}-71 \mathrm{~K}\right)$ and $95 \mathrm{~K}$. The solid line is a Gaussian fit to the data.

normal state spin gap increases in magnitude, and the intensity of the low energy fluctuations is suppressed.

To further determine the magnitude of the spin gap in the superconducting state, we carried out high-resolution measurements with the neutron final energy fixed at $E_{f}=14.78 \mathrm{meV}$. Figures $18(\mathrm{a}-\mathrm{c})$ show constant-energy scans at $\hbar \omega=24,30$, and $39 \mathrm{meV}$. The difference spectra at $\hbar \omega=24$ and $30 \mathrm{meV}$ between low and high temperatures are shown in Figs. 18(d-f). Careful temperature dependent measurements suggest that the weak structure marked by arrows in the low temperature raw data of Figs. 18(a) and (b) is not magnetic in origin. The opening of the spin gap at $\hbar \omega=30 \mathrm{meV}$ in the superconducting state is demonstrated by the difference spectra of Figs. 18(e) and (f), thus confirming the result of Fig. 17.

Finally, we present results on the incommensurate spin fluctuations at energies just below the resonance in $\mathrm{YBa}_{2} \mathrm{Cu}_{3} \mathrm{O}_{6.8}$. These high-resolution measurements were carried out on the HB-1 triple-axis spectrometer with $E_{f}=13.6 \mathrm{meV}$. Following the procedures established above, we show in Fig. 19 the temperature difference spectra at $\hbar \omega=32,34,37$, and $39 \mathrm{meV}$ above and below $T_{c}$. Incommensurate spin fluctuations were
FIG. 15: Difference spectra in constant-q scans at $(0.5,1.5,-$ 1.8) rlu above and below $T_{c}$ for $\mathrm{YBa}_{2} \mathrm{Cu}_{3} \mathrm{O}_{6.8}$ with the sample in the $(h, 3 h, l)$ zone. (a) The temperature difference between $95 \mathrm{~K}\left(T_{c}+13 \mathrm{~K}\right)$ and $150 \mathrm{~K}$. The positive scattering around $40 \mathrm{meV}$ again confirms the presence of the precursor for the resonance in the normal state. (b) The temperature difference between $11 \mathrm{~K}\left(T_{c}-71 \mathrm{~K}\right)$ and $95 \mathrm{~K}$. The solid line is a Gaussian fit to the data.

observed below $T_{c}$ at $\hbar \omega=32,34$, and $37 \mathrm{meV}$. As in the case of $\mathrm{YBa}_{2} \mathrm{Cu}_{3} \mathrm{O}_{6.6}$, the incommensurability of these fluctuations decreases with increasing energy and becomes commensurate at the resonance energy. In addition, the incommensurability of $\mathrm{YBa}_{2} \mathrm{Cu}_{3} \mathrm{O}_{6.8}$ at $\hbar \omega \approx 34$ $\mathrm{meV}$ is essentially the same as in the more underdoped $\mathrm{YBa}_{2} \mathrm{Cu}_{3} \mathrm{O}_{6.6}$ at $24 \mathrm{meV}$ [Fig. 10(a)]. However, the normal state spin fluctuations of $\mathrm{YBa}_{2} \mathrm{Cu}_{3} \mathrm{O}_{6.8}$ are clearly commensurate and centered around $(\pi, \pi)$ whereas the fluctuations in $\mathrm{YBa}_{2} \mathrm{Cu}_{3} \mathrm{O}_{6.6}$ show a flattish top indicative of the incommensurability at temperatures above $T_{c}$. Therefore, the spin fluctuation spectrum in $\mathrm{YBa}_{2} \mathrm{Cu}_{3} \mathrm{O}_{6.8}$ has a gap in the normal state $(\hbar \omega \approx 16 \mathrm{meV})$, a superconducting gap $(\hbar \omega \approx 30 \mathrm{meV})$, a resonance at $\hbar \omega \approx 39$ $\mathrm{meV}$, and commensurate-to-incommensurate transition for spin fluctuations below the resonance on entering the superconducting state.

\section{4. $\mathrm{YBa}_{2} \mathrm{Cu}_{3} \mathrm{O}_{6.93}$ and $\mathrm{YBa}_{2} \mathrm{Cu}_{3} \mathrm{O}_{6.95}$}

In the studies of ideally doped and overdoped $\mathrm{YBa}_{2} \mathrm{Cu}_{3} \mathrm{O}_{6+x}$, the important issues are the nature of 


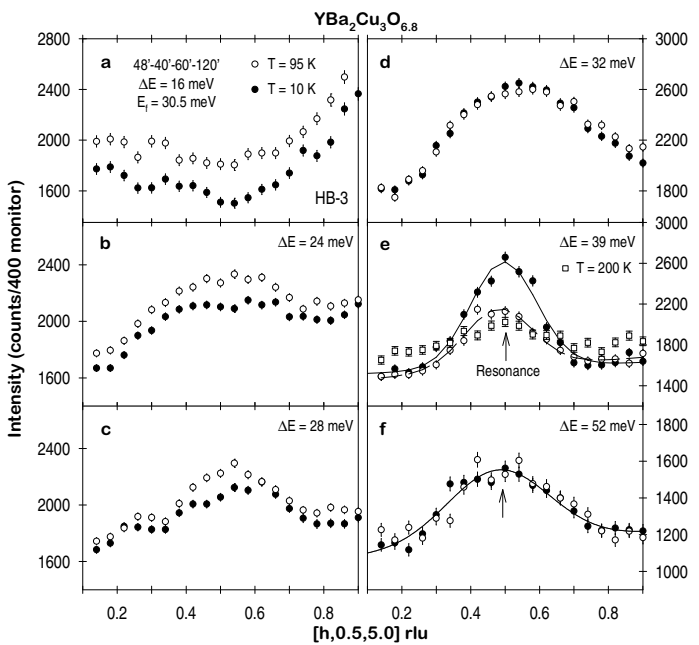

Figure 16
Dai et al.

FIG. 16: Raw constant-energy scans along the $[h, 0.5,5.0]$ direction for $\mathrm{YBa}_{2} \mathrm{Cu}_{3} \mathrm{O}_{6.8}$ in the low-resolution measurements with $E_{f}=30.5 \mathrm{meV}$. The energy transfers are $\hbar \omega=16 \mathrm{meV}$ (a), $24 \mathrm{meV}$ (b), $28 \mathrm{meV}$ (c), $32 \mathrm{meV}$ (d), $39 \mathrm{meV}$ (e), and $52 \mathrm{meV}(\mathrm{f})$. The resonance is clearly seen at $\hbar \omega=39 \mathrm{meV}$.

the normal state magnetic scattering and the evolution of the incommensurate spin fluctuations. To determine whether there is detectable magnetic scattering around $\hbar \omega \approx 40 \mathrm{meV}$ in the normal state of $\mathrm{YBa}_{2} \mathrm{Cu}_{3} \mathrm{O}_{6.93}$ and $\mathrm{YBa}_{2} \mathrm{Cu}_{3} \mathrm{O}_{6.95}$, we performed a series of constant- $Q$ scans at $(\pi, \pi)$ at various temperatures below and above $T_{c}$. Figures 20 and 21 display the low temperature minus high temperature difference spectra for $\mathrm{YBa}_{2} \mathrm{Cu}_{3} \mathrm{O}_{6.93}$ and $\mathrm{YBa}_{2} \mathrm{Cu}_{3} \mathrm{O}_{6.95}$, respectively. In both compounds, we find the resonance peak at $\hbar \omega \approx 40 \mathrm{meV}$ below $T_{c}$ and no trace or reminiscence of the resonance above $T_{c}$ as seen in all of the underdoped materials. Careful constantenergy scans for $\mathrm{YBa}_{2} \mathrm{Cu}_{3} \mathrm{O}_{6.95}$ at $\hbar \omega=28,30,32$ and $40 \mathrm{meV}$ are shown in Figs. 22(a-c). The temperature difference spectra in Figs. 22(d-f) yield a spin gap value of $\hbar \omega=32 \mathrm{meV}$ in the superconducting state and show no discernible normal state magnetic scattering [16].

To further elucidate the nature of the incommensurate spin fluctuations, we performed a series of constantenergy scans at energies just below the $40 \mathrm{meV}$ resonance for highly doped $\mathrm{YBa}_{2} \mathrm{Cu}_{3} \mathrm{O}_{6.95}$. Figure 23 summarizes the outcome below and above $T_{c}$ at $\hbar \omega=34$, 35.5, 37 and $40 \mathrm{meV}$. Clear incommensurate spin fluctuations were found at $\hbar \omega=34,35.5$, and $37 \mathrm{meV}$ in the low temperature superconducting state. Surprisingly, the incommensurability of the fluctuations at $\hbar \omega=34$

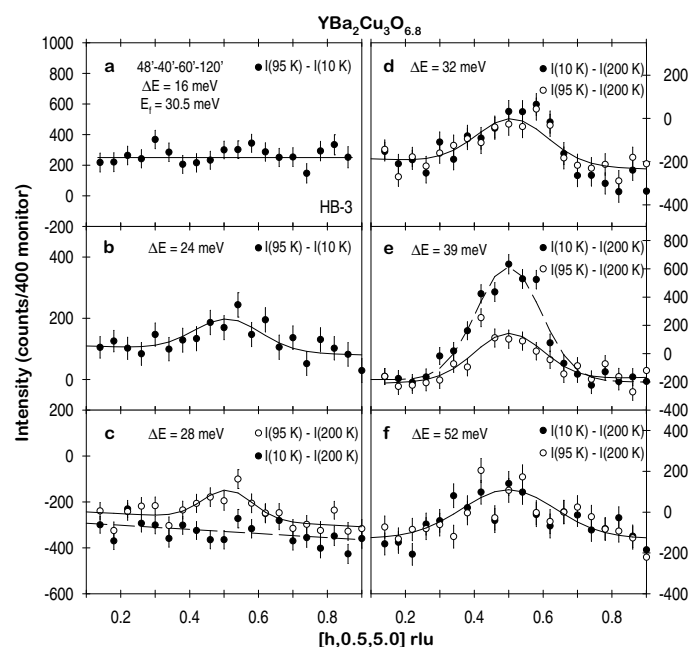

Figure 17
Dai et al.

FIG. 17: Difference spectra in constant-energy scans along the $[h, 0.5,5.0]$ direction for $\mathrm{YBa}_{2} \mathrm{Cu}_{3} \mathrm{O}_{6.8}$. The energy transfers are $\hbar \omega=16 \mathrm{meV}$ (a), $24 \mathrm{meV}$ (b), $28 \mathrm{meV}$ (c), $32 \mathrm{meV}$ (d), $39 \mathrm{meV}$ (e), $52 \mathrm{meV}$ (f). (a) suggests no change in susceptibility between the normal and superconducting states. (b) and (c) indicate that Gaussian-like normal state scattering is suppressed in the superconducting state at $\hbar \omega=24$ and $28 \mathrm{meV}$. The situation for $\hbar \omega=32 \mathrm{meV}$ is not clear in this low-resolution measurement although there are clear magnetic intensities in the normal and the superconducting states. The intensity gain of the resonance is seen in (e), and the magnetic scattering appears to have weak temperature dependence at $52 \mathrm{meV}$ (f). The solid lines are Gaussian fits to the data.

$\mathrm{meV}$ is $\delta=0.11 \pm 0.0128 \mathrm{rlu}$, which is indistinguishable from all the other underdoped compounds with $x \geq 0.6$. The incommensurability changes to $\delta=0.089 \pm 0.008$ and $0.084 \pm 0.02 \mathrm{rlu}$ for $\hbar \omega=35.5 \mathrm{meV}$ and $37 \mathrm{meV}$, respectively. Although there are weak features in the constant-energy scans just above $T_{c}$ [Figs. 23(a-d)], careful temperature dependent studies of the constant-energy profiles indicate that these features are nonmagnetic in origin [Fig. 23(e), open circles]. Thus, incommensurate fluctuations in highly doped $\mathrm{YBa}_{2} \mathrm{Cu}_{3} \mathrm{O}_{6+x}$ appear only in the low temperature superconducting state at energies close to the resonance. Furthermore, the incommensurabilities of the low energy spin fluctuations are insensitive to the doping for $\mathrm{YBa}_{2} \mathrm{Cu}_{3} \mathrm{O}_{6+x}$ with $x \geq 0.6$ and saturate at $\delta=1 / 10$. In contrast, incommensurate spin fluctuations in the single-layer $\mathrm{La}_{2-x} \mathrm{Sr}_{x} \mathrm{CuO}_{4}$ near optimal doping survive to temperatures well above $T_{c}$ [49], and the incommensurabilities of the low energy spin fluctua- 


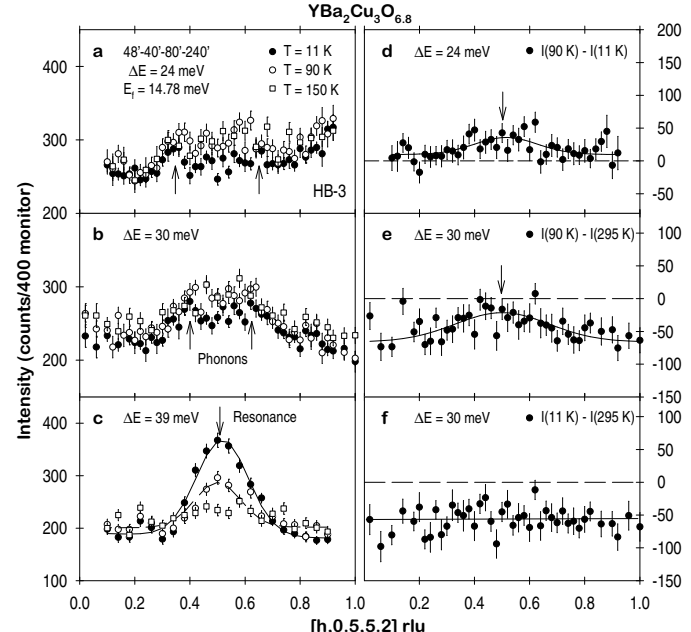

Figure 18

FIG. 18: High-resolution constant-energy scans along the $[h, 0.5,5.2]$ direction for $\mathrm{YBa}_{2} \mathrm{Cu}_{3} \mathrm{O}_{6.8}$. The energy transfers are $\hbar \omega=24 \mathrm{meV}$ (a), $30 \mathrm{meV}$ (b), and $39 \mathrm{meV}$ (c). The weak structures in (a) and (b) at $11 \mathrm{~K}$ marked by the arrows are nonmagnetic scattering. (d) The difference spectrum for $\hbar \omega=24 \mathrm{meV}$ between $90 \mathrm{~K}$ and $11 \mathrm{~K}$ shows a Gaussian profile in the normal state, consistent with Fig. 17(b). (e) and (f) demonstrate the opening of the spin gap from the normal to the superconducting state at $\hbar \omega=30 \mathrm{meV}$. No evidence for incommensurate spin fluctuations is found at $\hbar \omega=30 \mathrm{meV}$. The solid lines are Gaussian fits to the data and guides to the eye.

tions increase smoothly to the saturation value of $\delta=1 / 8$ for materials with optimal doping [8].

\section{B. Discussion}

\section{Doping dependence of the incommensurability}

The existence of incommensurate spin fluctuations now appears to be a common feature of the $\mathrm{YBa}_{2} \mathrm{Cu}_{3} \mathrm{O}_{6+x}$ family of superconductors [50]. Such fluctuations have also been observed in $\mathrm{Sr}^{2+}$ - and oxygen- doped samples of $\mathrm{La}_{2} \mathrm{CuO}_{4}$ [11]. Thus, it becomes clear that incommensurability is the common feature between these two classes of the most studied superconductors. However, before attempting any quantitative comparison of the similarities between $\mathrm{YBa}_{2} \mathrm{Cu}_{3} \mathrm{O}_{6+x}$ and $\mathrm{La}_{2-x} \mathrm{Sr}_{x} \mathrm{CuO}_{4}$, we must first consider the number of holes $(p)$ in the $\mathrm{CuO}_{2}$ plane in these two systems. For $\mathrm{La}_{2-x} \mathrm{Sr}_{x} \mathrm{CuO}_{4}$,

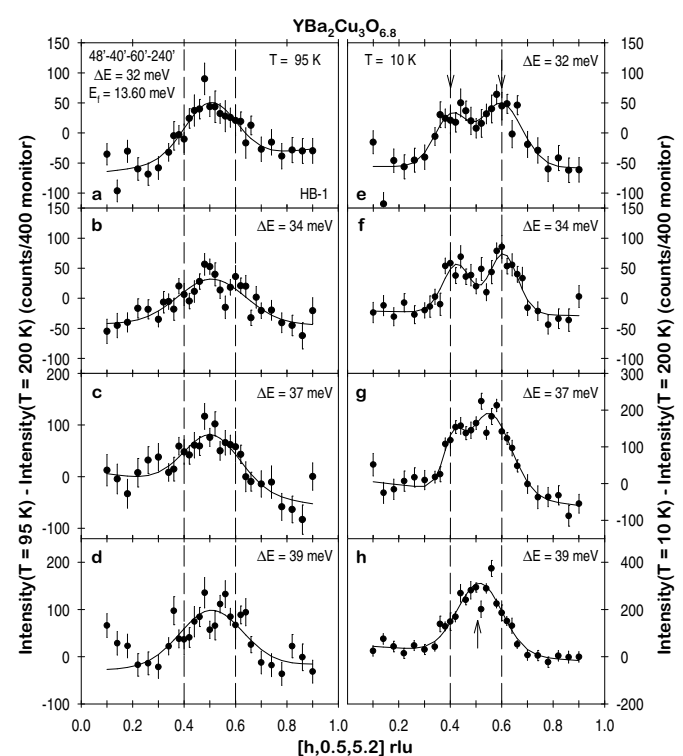

Figure 19
Dai et al.

FIG. 19: Difference spectra in high-resolution constantenergy scans along the $[h, 0.5,5.2]$ direction above and below $T_{c}$ for $\mathrm{YBa}_{2} \mathrm{Cu}_{3} \mathrm{O}_{6.8}$. The energy transfers are $\hbar \omega=32$ $\mathrm{meV}$ (a) and (e), $34 \mathrm{meV}$ (b) and (f), $37 \mathrm{meV} \mathrm{(c)} \mathrm{and} \mathrm{(g),}$ and $39 \mathrm{meV}(\mathrm{d})$ and $(\mathrm{h})$. The scattering below the $39 \mathrm{meV}$ resonance changes from commensurate in the normal state to incommensurate in the superconducting state. Similar behavior have been reported by Bourges et al. for $\mathrm{YBa}_{2} \mathrm{Cu}_{3} \mathrm{O}_{6.85}$ [51]. The solid lines are Gaussian fits to the data.

Yamada and coworkers [8] found an excellent linear relationship between $\delta$ and the effective hole concentration up to around $p \approx 0.12$. Moreover, the incommensurability $\delta$ is energy independent for $\hbar \omega<15 \mathrm{meV}$ [6]. The situation for $\mathrm{YBa}_{2} \mathrm{Cu}_{3} \mathrm{O}_{6+x}$ is more subtle. Using the established approximate parabolic relationship $T_{c} / T_{c, \max }=1-82.6(p-0.16)^{2}$, where $T_{c, \max }$ is the $\max -$ imum transition temperature of the system, and $p$ is the hole-doping 45], we can calculate the effective hole doping $p$ of $\mathrm{YBa}_{2} \mathrm{Cu}_{3} \mathrm{O}_{6+x}$ from their $T_{c}$ values. However, as shown in Figs. 5, 10, 19, and 23, the incommensurability in $\mathrm{YBa}_{2} \mathrm{Cu}_{3} \mathrm{O}_{6+x}$ decreases with increasing energy close to the resonance. As a consequence, it is difficult to compare directly the doping dependence of the incommensurability in these two families of materials as $\delta$ is not a well defined quantity for $\mathrm{YBa}_{2} \mathrm{Cu}_{3} \mathrm{O}_{6+x}$. In order to make such comparison, we chose to systematically use the $\delta$ for the incommensurate low frequency spin fluctuations furthest away in energy from the resonace.

In Figure 24, we plot the incommensurability $\delta$ obtained in such procedure as a function of $p$. For completeness, we also included the data of Arai et al. (open 


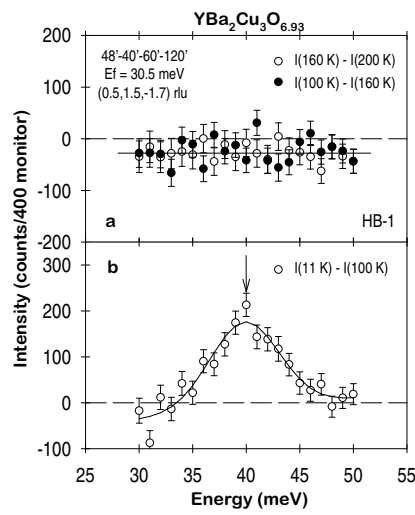

Figure 20
Dai et al.

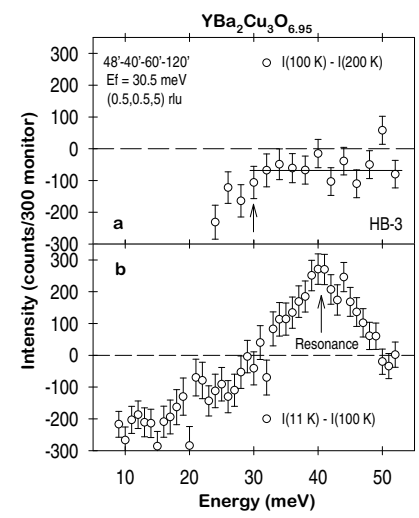

Figure 21
FIG. 20: Difference spectra in constant-q scans at $(0.5,1.5,-$ 1.7) rlu above and below $T_{c}$ for $\mathrm{YBa}_{2} \mathrm{Cu}_{3} \mathrm{O}_{6.93}$ with the crystal in the $(h, 3 h, l)$ zone. (a) The filled circles show the difference between $100 \mathrm{~K}\left(T_{c}+7.5 \mathrm{~K}\right)$ and $160 \mathrm{~K}$. The open circles are the difference between $160 \mathrm{~K}$ and $200 \mathrm{~K}$. Note that there is no positive scattering around $40 \mathrm{meV}$ in the normal state, which suggests no precursor of the resonance. (b) The temperature difference between $11 \mathrm{~K}\left(T_{c}-81.5 \mathrm{~K}\right)$ and $100 \mathrm{~K}$. The solid line in (b) is a Gaussian fit to the data.

square) for $\mathrm{YBa}_{2} \mathrm{Cu}_{3} \mathrm{O}_{6.7}$ 25 and very recent data of Bourges et al. (filled square) for $\mathrm{YBa}_{2} \mathrm{Cu}_{3} \mathrm{O}_{6.85}$ [51]. As we can see from the figure, the incommensurability increases initially with doping, but it saturates quickly at $\delta \approx 0.1$ for hole doping $p \geq 0.1$. This result is different from $\mathrm{La}_{2-x} \mathrm{Sr}_{x} \mathrm{CuO}_{4}$ where the incommensurability is found to be linear with $\delta$ until reaching 0.125 at large doping [8]. For $\mathrm{La}_{2-x} \mathrm{Sr}_{x} \mathrm{CuO}_{4}$, the saturation of incommensurability above $x \approx 0.12$ appears to be related to the saturation of the density of $\mathrm{O}(2 p)$-type holes in the $\mathrm{CuO}_{2}$ plane as suggested from optical measurements [52]. In the case of $\mathrm{YBa}_{2} \mathrm{Cu}_{3} \mathrm{O}_{6+x}$, the incommensurability saturates for $x \geq 0.6$ whereas the hole concentration $p$ in the $\mathrm{CuO}_{2}$ plane continues to increase with increasing oxygen doping and $T_{c}$ 45. Therefore, the saturation of the incommensurability in $\mathrm{YBa}_{2} \mathrm{Cu}_{3} \mathrm{O}_{6+x}$ may have a different microscopic origin from that of $\mathrm{La}_{2}-x \mathrm{Sr}_{x} \mathrm{CuO}_{4}$.

Although it is interesting to compare the incommensurability as a function of effective hole concentration in the $\mathrm{CuO}_{2}$ plane of $\mathrm{YBa}_{2} \mathrm{Cu}_{3} \mathrm{O}_{6+x}$ and $\mathrm{La}_{2-x} \mathrm{Sr}_{x} \mathrm{CuO}_{4}$, a more robust test of the similarities in these two families of materials can be made by comparing the relation between
FIG. 21: Difference spectra in constant-q scans at $(0.5,0.5,5)$ rlu above and below $T_{c}$ for $\mathrm{YBa}_{2} \mathrm{Cu}_{3} \mathrm{O}_{6.95}$. (a) The difference spectrum between $100 \mathrm{~K}\left(T_{c}+8 \mathrm{~K}\right)$ and $200 \mathrm{~K}$. Note that there are no observable features above the background around 40 $\mathrm{meV}$ in the normal state, again confirming no precursor of the resonance. (b) The temperature difference between $11 \mathrm{~K}$ $\left(T_{c}-81 \mathrm{~K}\right)$ and $100 \mathrm{~K}$.

$T_{c}$ and $\delta$ because these two quantities are free from any uncertainties associated with the doping level or oxygen stoichiometry. For $\mathrm{La}_{2-x} \mathrm{Sr}_{x} \mathrm{CuO}_{4}$, Yamada et al. found a linear relationship between $T_{c}$ and $\delta$ up to the optimal doping regime [8]. Using $T_{c}=\hbar \nu_{214}^{*} \delta$, Balatsky and Bourges 43] extracted a velocity $\hbar \nu_{214}^{*}=20 \mathrm{meVÅ.} \mathrm{For}$ $\mathrm{YBa}_{2} \mathrm{Cu}_{3} \mathrm{O}_{6+x}$, these authors suggested a similar linear relation between $T_{c}$ and the $q$ width in momentum space, $\Delta q$ (HWHM), for the whole doping range: $T_{c}=\hbar \nu^{*} \Delta q$, with $\hbar \nu^{*}=35 \mathrm{meV} \AA$. They then claimed strong similarities in the magnetic states of $\mathrm{YBa}_{2} \mathrm{Cu}_{3} \mathrm{O}_{6+x}$ and $\mathrm{La}_{2-x} \mathrm{Sr}_{x} \mathrm{CuO}_{4}$ 43]. In Fig. 25, we plot the relation between $T_{c}$ and the measured incommensurability $\delta$ for $\mathrm{YBa}_{2} \mathrm{Cu}_{3} \mathrm{O}_{6+x}$ in the whole doping range. For superconducting transition temperatures below $\sim 60 \mathrm{~K}$, our data are consistent with the proposed linear relation between $T_{c}$ and $\delta$ with $\hbar \nu^{*}=36.6 \mathrm{meV} \AA$ [43]. However, the incommensurability is essentially unchanged for all samples with $T_{c}$ larger than $60 \mathrm{~K}$. Therefore, if there were excitations associated with such a velocity, its magnitude would approach infinity for samples with transition temperatures above $60 \mathrm{~K}$. 


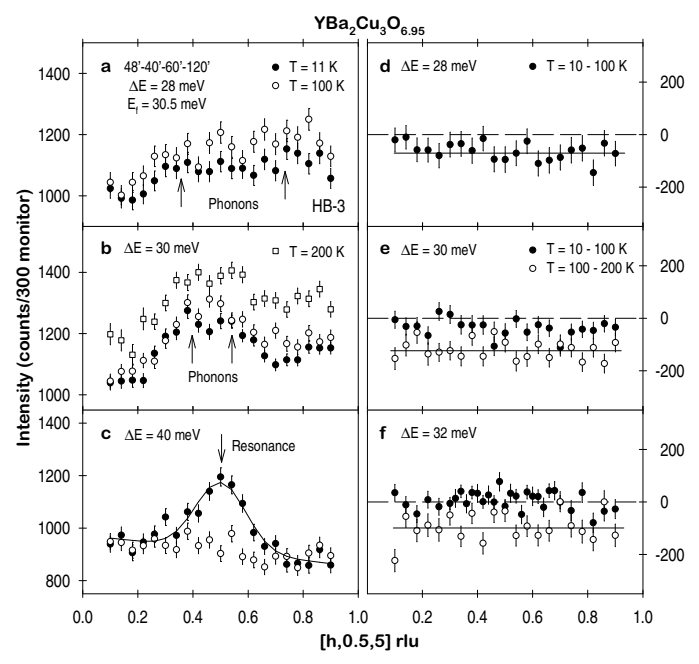

Figure 22

FIG. 22: Constant-energy scans along the $[h, 0.5,5]$ direction at various temperatures for $\mathrm{YBa}_{2} \mathrm{Cu}_{3} \mathrm{O}_{6.95}$. The energy transfers are $\hbar \omega=28 \mathrm{meV}$ (a), $30 \mathrm{meV}$ (b), and $40 \mathrm{meV}$ (c). The weak structures in (a) and (b) at $11 \mathrm{~K}$ marked by arrows are nonmagnetic scattering. (d) The temperature difference spectrum for $\hbar \omega=28 \mathrm{meV}$ between $10 \mathrm{~K}$ and $100 \mathrm{~K}$ shows no observable feature around $(\pi, \pi)$. (e) and (f) suggest no discernible magnetic scattering above and below $T_{c}$ at $\hbar \omega=30$ and $32 \mathrm{meV}$. In addition, no evidence for incommensurate spin fluctuations is found below $\hbar \omega=32 \mathrm{meV}$. The solid lines are Gaussian fits to the data and guides to the eye.

\section{Doping dependence of the spin gap in the superconducting state}

One of the salient features of the spin excitations spectra in $\mathrm{YBa}_{2} \mathrm{Cu}_{3} \mathrm{O}_{6+x}$ is the presence of spin gaps in the normal and superconducting states. For example, the low-energy spin fluctuations of $\mathrm{YBa}_{2} \mathrm{Cu}_{3} \mathrm{O}_{6.8}$ show well defined Gaussian peaks centered around $(\pi, \pi)$ at energies above $24 \mathrm{meV}$ in the normal state. On cooling the system to below $T_{c}$, the normal state scattering is suppressed which indicates the opening of a gap in the spin fluctuation spectrum (Figs. 17 and 18). In Figure 26, we plot the magnitude of the spin gap in the low temperature superconducting state as a function of $T_{c}$ for the whole doping range. The solid line shows the linear relation between $T_{c}$ and the magnitude of the spin gap $\left(E_{s g}\right)$ up to the optimal doping with $E_{s g} / k_{B} T_{c}=3.8$. This empirical result connects directly the spin gap and the superconductivity. The value of $E_{s g} / k_{B} T_{c}=3.8$ is not far away from the $2 \Delta / k_{B} T_{c}=3.52$ for the weak-coupling

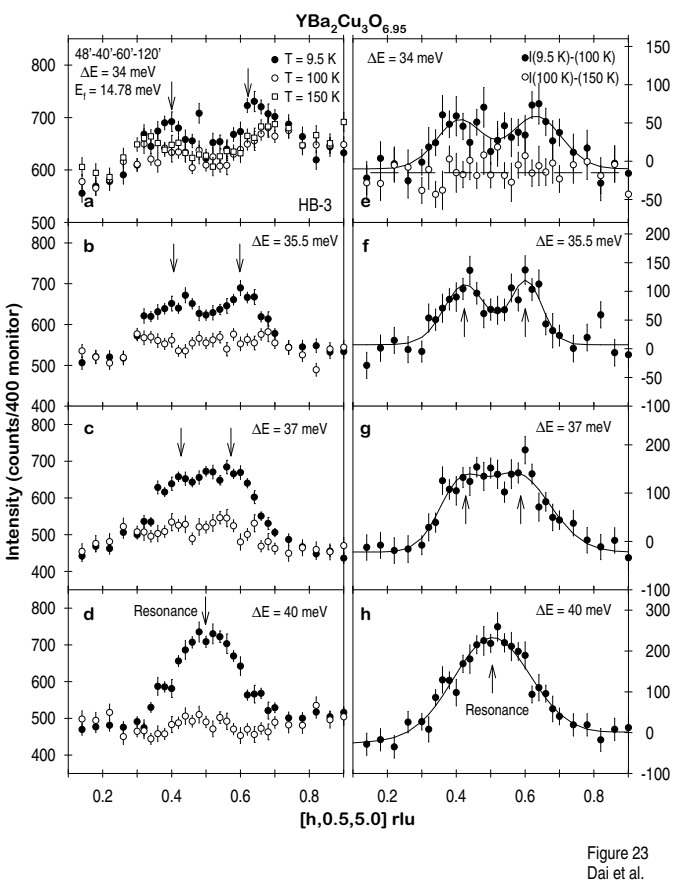

FIG. 23: High-resolution constant-energy scans along the $[h, 0.5,5]$ direction at various temperatures for $\mathrm{YBa}_{2} \mathrm{Cu}_{3} \mathrm{O}_{6.95}$. The energy transfers are $\hbar \omega=34 \mathrm{meV}$ (a), $35.5 \mathrm{meV}$ (b), 37 $\mathrm{meV}(\mathrm{c})$, and $40 \mathrm{meV}(\mathrm{d})$. The enhancement of the scattering from $100 \mathrm{~K}$ to $9.5 \mathrm{~K}$ is marked by arrows. (e) The filled circles show the difference between $9.5 \mathrm{~K}\left(T_{c}-82.5 \mathrm{~K}\right)$ and $100 \mathrm{~K}$. The open circles are the difference between $100 \mathrm{~K}$ and $150 \mathrm{~K}$. Therefore, while there is no discernible magnetic intensity in the normal state, clear incommensurate spin fluctuations are observed in the superconducting state. (f) and (g) show the presence of incommensurate magnetic scattering at $\hbar \omega=35.5$ and $37 \mathrm{meV}$ with the incommensurability marked by arrows. (h) shows the commensurate resonance. The solid and dashed lines are Gaussian fits to the data and guides to the eye, respectively.

limit of the BCS theory of superconductivity, where $\Delta$ is the superconducting gap. For samples with low oxygen doping and low $T_{c}$, the linear relation between $T_{c}$ and $E_{s g}$ breaks down, possibly due to the oxygen doping inhomogeneity and broad superconducting transitions in weakly-doped materials.

\section{Doping dependence of the resonance and superconducting condensation energy}

Among the many interesting features observed in the spin excitations spectra of $\mathrm{YBa}_{2} \mathrm{Cu}_{3} \mathrm{O}_{6+x}$, perhaps the most-discussed is the collective acoustic spin excitation named the "resonance". First reported by RossatMignod et al. 12 for $\mathrm{YBa}_{2} \mathrm{Cu}_{3} \mathrm{O}_{6.92}$, the resonance at $\sim 40 \mathrm{meV}$ in the highly doped $\mathrm{YBa}_{2} \mathrm{Cu}_{3} \mathrm{O}_{6+x}$ was shown 


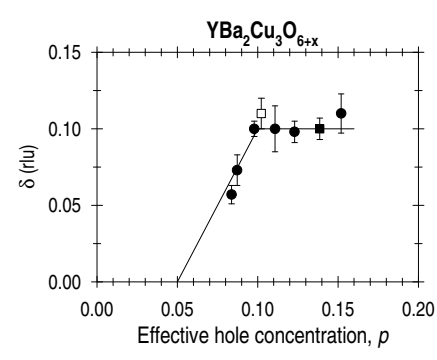

Figure 24
Dai et al

FIG. 24: The incommensurability $\delta$ as a function of effective hole concentration, $p$, calculated from the parabolic relationship discussed in the text. $\delta$ is seen to saturate for $p \geq 0.1$. The open and filled squares are from Ref. [25] and [51], respectively. The filled circles are from the present work. The solid lines are guides to the eye. Note that superconductivity in cuprates occurs for $p \geq 0.05$ [44].

to be magnetic in origin, narrow in energy 14, and to appear exclusively below $T_{c}$ [16]. In subsequent experiments 19, 20, 21], the resonance was also found in underdoped $\mathrm{YBa}_{2} \mathrm{Cu}_{3} \mathrm{O}_{6+x}$ with the resonance energy $\left(E_{r}\right)$ approximately scaling with $T_{c}: E_{r} \propto k_{B} T_{c}$. Consistent with previous results [43], the coherence length of the resonance $(\xi)$ is weakly doping dependent except for $\mathrm{YBa}_{2} \mathrm{Cu}_{3} \mathrm{O}_{6.6}$ which has considerably larger $\xi$ than for all the other dopings (Fig. 27). The coherence length of the incommensurate spin fluctuations (Fig. 28) also appears to be weakly doping dependent. In addition, we note that on approaching the optimal oxygen doping for $\mathrm{YBa}_{2} \mathrm{Cu}_{3} \mathrm{O}_{6+x}$, the resonance energy $\left(E_{r}\right)$ is insensitive to the changing $T_{c}$ (Fig. 29). Therefore, the linear relationship between $E_{r}$ and $T_{c}$ appears to break down for materials with oxygen concentrations close to its optimal doping. Finally, we compared the peak intensity of the lowest-energy observable incommensurate fluctuations with that of the resonance (Fig. 30). While the ratio clearly decreases with increasing doping for $p \leq 0.1$, it saturates to a value of $\sim 0.25$ for $p \geq 0.1$.

One interesting aspect of the resonance peak is its unusual temperature dependence. For optimal $(x=0.93)$ and over doped $(x=1)$ samples, the onset of the res-

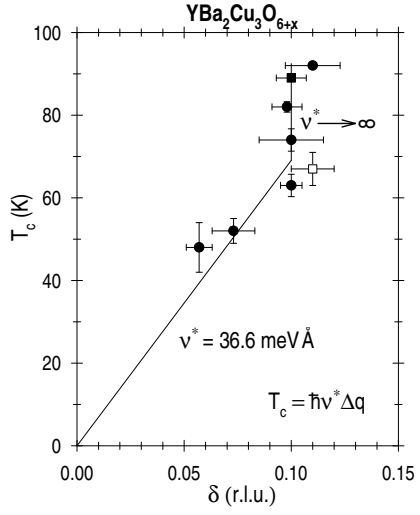

Figure 25
Dai et al.

FIG. 25: The incommensurability $\delta$ plotted as a function of $T_{c}$. The solid line for $T_{c} \leq 63 \mathrm{~K}$ is a fit using $T_{c}=\hbar \nu^{*} \delta$ with $\hbar \nu^{*} \approx 36.6 \mathrm{meVÅ}$. For $T_{c} \geq 63 \mathrm{~K}, \delta$ saturates to $0.1 \mathrm{rlu}$ thus giving $\nu^{*} \longrightarrow \infty$.

onance occurs at a temperature $T^{*}$ which almost coincides with $T_{c}$ 14. For underdoped $x=0.8$ and 0.6, $T^{*}$ increases to temperatures well above $T_{c}$ while both $T_{c}$ and the resonance energy itself are reduced [27]. By empirically comparing the cross-over temperature $T^{*}$ and the pseudogap temperature obtained by other techniques 53, 54, 55, we associate the initial occurrence of the resonance with the pseudogap temperature [27]. In recent published [56] and unpublished [57] papers, Bourges et al. [57] claimed that there is no justification for a separation of the normal state spin excitations spectrum into resonant and nonresonant parts. Although these authors agreed that the broad maximum of the spin susceptibility in the normal state occurs at the same energy as the resonance peak in some underdoped materials 27, 58, they argued that "the apparent equivalence of the normal state energy and the resonance peak energy breaks down in underdoped samples closer to optimal doping" [57.

Here we point out that the claims by Bourges et al. are incorrect. As can be seen from our results in Sec. IIIA1-3, there are clear enhancements in the spin susceptibility around the resonance energy at temperatures above $T_{c}$ for all the underdoped compounds including $\mathrm{YBa}_{2} \mathrm{Cu}_{3} \mathrm{O}_{6.8}$. These observations are in sharp contrast to the claims of Bourges et al. 57. Moreover, from the temperature dependence of the resonance intensity 


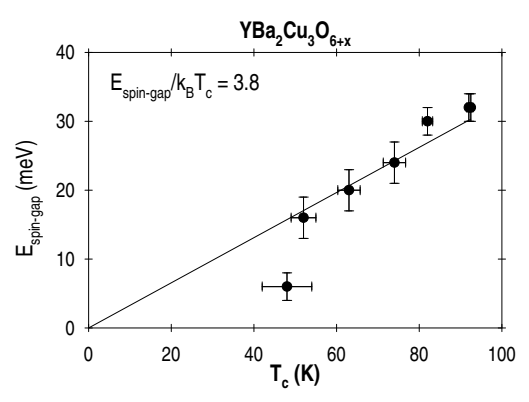

Figure 26

FIG. 26: The magnitude of the spin-gap in the superconducting state as a function of $T_{c}$. The solid line gives $E_{s g} / k_{B} T_{c}=3.8$. For oxygen concentrations $x$ below 0.45 , the linear relationship between $E_{s g}$ and $k_{B} T_{c}$ breaks down.

as a function of doping [27, we find that the intensity of the resonance changes smoothly and shows progressively larger pretransitional regime above $T_{c}$ as a function of decreasing doping. As a consequence, it is natural to associate the enhancement of the spin susceptibility above $T_{c}$ around the resonance energy in underdoped materials as the precursor of the resonance.

We also emphasize that the temperature dependence of the resonance [27] is closely related to the thermodynamics of $\mathrm{YBa}_{2} \mathrm{Cu}_{3} \mathrm{O}_{6+x}$ near $T_{c}$ [59] and hence the superconducting condensation energy $\mid 60,61,62,663$. If the electronic entropy changes around $\bar{T}_{c}$ are mostly due to spin excitations [27, they must respond to an external magnetic field in qualitatively the same way [64]. In a conventional type-II BCS superconductor [65], the effect of a field is to simply shift the superconducting specific heat anomaly $\left(C^{e l}(x, T)\right)$ to lower temperatures and to reduce its amplitude until the upper critical field, $B_{c 2}(0)$, is reached [66]. For high- $T_{c}$ superconductors such as $\mathrm{YBa}_{2} \mathrm{Cu}_{3} \mathrm{O}_{6+x}$, the electronic specific heat anomaly near $T_{c}$ is drastically suppressed by a moderate field along the $c$-axis while it is little affected by the same field parallel to the $a b$-plane $[67$.

In a recent experiment on $\mathrm{YBa}_{2} \mathrm{Cu}_{3} \mathrm{O}_{6.6}$ [68], we discovered that a moderate magnetic field $(B=6.8 \mathrm{~T})$ along the direction approximately perpendicular to the $\mathrm{CuO}_{2}$ planes ( $c$-axis) can drastically suppress the intensity of

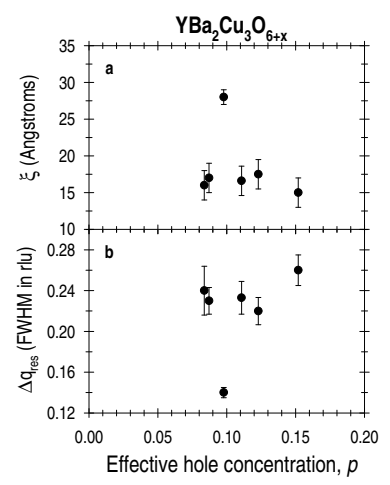

Figure 27 Dai et al.

FIG. 27: The minimum coherence length and dynamical $q$ width (FWHM) of the resonance as a function of effective hole concentration, $p$. At present, the origin of the anomalous long coherence length of the resonance for $\mathrm{YBa}_{2} \mathrm{Cu}_{3} \mathrm{O}_{6.6}$ is unclear. The instrumental resolutions along the scan direction are $\sim 0.12 \mathrm{rlu}$ for the $\mathrm{PG}(002)$ monochromator and 0.07 rlu for the $\mathrm{Be}(002)$ monochromator at $\hbar \omega=34 \mathrm{meV}$. Slightly different values are obtained at other energies. Since the instrumental resolution is much smaller than the observed $q$ width of the resonance shown in (b), the resonance coherence lengths in (a) are mostly intrinsic and not resolution limited.

the resonance at $34 \mathrm{meV}$ while leaving other scattering relatively unaltered. Similar, but much smaller effect also occurs for the same field parallel to the $\mathrm{CuO}_{2}$ planes. Our results thus provide further support for the intimate connection between the magnetic excitation spectrum and thermodynamics of high- $T_{c}$ superconductors [27]. The persistence of a field effect above $T_{c}$ is consistent with the precursor of the resonance in the normal state of the underdoped $\mathrm{YBa}_{2} \mathrm{Cu}_{3} \mathrm{O}_{6+x}$.

\section{Stripes versus Fermi-liquid}

Fermi-liquid theory, based on the premise that electron correlation effects in the metallic state of matter correspond to the noninteracting "quasiparticles" on a Fermi surface, has been successful in solving many of the important problems in condensed-matter physics, including conventional superconductivity. In the case of copperoxides, where high- $T_{c}$ superconductivity is obtained by 


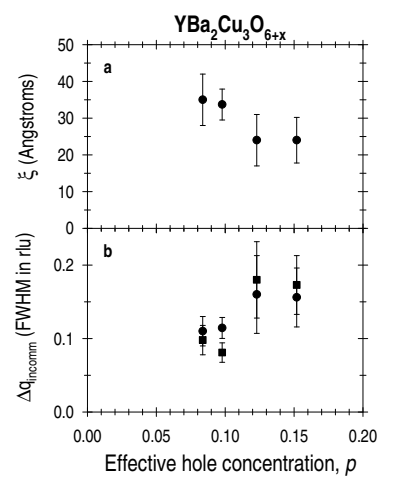

Figure 28
Dai et al.

FIG. 28: The minimum coherence length and dynamical $q$ width (FWHM) of the incommensurate spin fluctuations as a function of effective hole concentration, $p$, for $\mathrm{YBa}_{2} \mathrm{Cu}_{3} \mathrm{O}_{6+x}$ with $x=0.45$ at $\hbar \omega=24 \mathrm{meV}, 0.6$ at $24 \mathrm{meV}, 0.8$ at 32 $\mathrm{meV}, 0.93$ at $34 \mathrm{meV}$. The filled circles and squares in (b) are the measured widths for the left and right incommensurate peaks, respectively. The instrumental resolutions along the scan direction are $\sim 0.09$ rlu for $\mathrm{PG}(002)$ monochromator and $0.049 \mathrm{rlu}$ for $\mathrm{Be}(002)$ monochromator at $\hbar \omega=24 \mathrm{meV}$. They change to $\sim 0.12$ rlu for $\hbar \omega=32$ and $34 \mathrm{meV}$ with PG(002) monochromator. Therefore, the slightly larger observed widths at $\hbar \omega=32$ and $34 \mathrm{meV}$ in (b) are due to the broadened instrumental resolution at these energies.

hole-doping of the AF insulating parent compounds, an important class of models [69, 70, 71, 72 argues that electrons in these materials cannot be described by quasiparticles and Fermi-liquid theory. Rather, the electrons have been incorporated in complex, inhomogeneous (fluctuating) patterns of the charge and spin confined to separate linear regions in the crystal resembling "stripes" [73. Since their discovery [3, 4, 5, 6, 7, 8, 9, 10, 11], the incommensurate spin fluctuations in the superconducting $\mathrm{La}_{2-x} \mathrm{Sr}_{x} \mathrm{CuO}_{4}$ have been interpreted as due to the presence of dynamic spin stripes [74, 75, 76, 77] or as the consequence of spin-flip scattering across a nested Fermi surface 788, 79, 80, 81, 82, 83, 84. In 1995, Tranquada and coworkers 46] found evidence supporting the former interpretation in a closely related material $\mathrm{La}_{1.88-y} \mathrm{Nd}_{y} \mathrm{Sr}_{0.12} \mathrm{CuO}_{4}$. For $\mathrm{Nd}$ substitution of $y=0.4$, the distortion of the crystal structure causes the condensation of the incommensurate spin fluctuations in Nd-free $\mathrm{La}_{2-x} \mathrm{Sr}_{x} \mathrm{CuO}_{4}$ into a spin density wave (SDW)

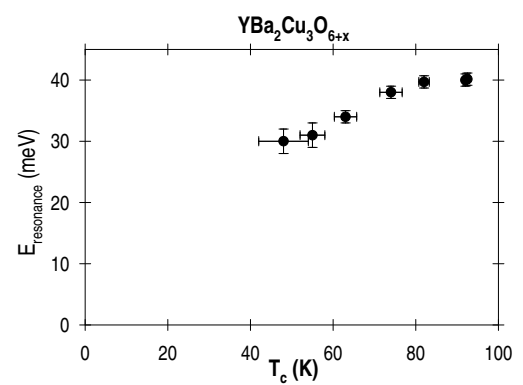

Figure 29
Dai et al.

FIG. 29: The resonance energy as a function of $T_{c}$ for samples used in this study. The horizontal error bars are the superconducting transition widths.

at the same wave vector 46. The analysis of the elastic magnetic SDW peaks and their corresponding chargeordering peaks associated with the modulation of the underlying lattice indicate the presence of a static striped phase in $\mathrm{La}_{1.88-y} \mathrm{Nd}_{y} \mathrm{Sr}_{0.12} \mathrm{CuO}_{4}$. This strongly suggests that the incommensurate fluctuations in superconducting $\mathrm{La}_{2-x} \mathrm{Sr}_{x} \mathrm{CuO}_{4}$ are a fluctuating version (dynamic) of the stripes.

However, if the stripe picture is universal for all high$T_{c}$ superconductors, it should also be able to explain the incommensurate spin fluctuations of $\mathrm{YBa}_{2} \mathrm{Cu}_{3} \mathrm{O}_{6+x}$. In the case of $\mathrm{La}_{2-x} \mathrm{Sr}_{x} \mathrm{CuO}_{4}$, the incommensurability saturates at " $1 / 8$ ". Whereas the incommensurability of the low-energy spin fluctuations in $\mathrm{YBa}_{2} \mathrm{Cu}_{3} \mathrm{O}_{6+x}$ follows the expected linear behavior as a function of $T_{c}$ for low doping materials [8] but abruptly saturates at " $1 / 10$ " for $x \geq 0.6$. This could mean that the minimum stripe spacing in $\mathrm{YBa}_{2} \mathrm{Cu}_{3} \mathrm{O}_{6+x}$ is five unit cells compared to four in $\mathrm{La}_{2-x} \mathrm{Sr}_{x} \mathrm{CuO}_{4}$ 46. However, the reduction of the incommensurability with increasing energy close to the resonance in $\mathrm{YBa}_{2} \mathrm{Cu}_{3} \mathrm{O}_{6+x}$, first reported by Arai et al. [25] and subsequently confirmed by Bourges et al. [51] in underdoped compounds, is different from the energy independent incommensurability of the low-frequency spin fluctuations in $\mathrm{La}_{2-x} \mathrm{Sr}_{x} \mathrm{CuO}_{4}$ [6]. In addition, the incommensurate fluctuations in $\mathrm{La}_{2-x} \mathrm{Sr}_{x} \mathrm{CuO}_{4}$ are observed at temperatures well above $T_{c}$ 49. Such fluctuations in $\mathrm{YBa}_{2} \mathrm{Cu}_{3} \mathrm{O}_{6+x}$ are present above $T_{c}$ for under- 


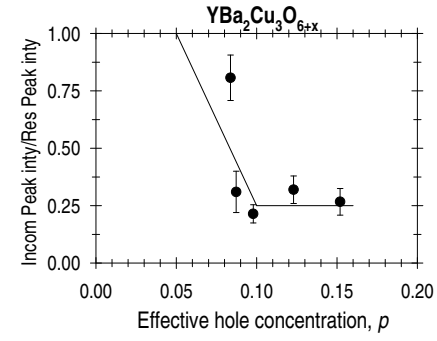

Figure 30
Dai et al

FIG. 30: The ratio between the peak intensity of incommensurate spin fluctuations and the resonance as a function of effective hole concentration, $p$, for $\mathrm{YBa}_{2} \mathrm{Cu}_{3} \mathrm{O}_{6+x}$. The intensity of incommensurate spin fluctuations is at $\hbar \omega=24 \mathrm{meV}$ for $x=0.45,0.5$, and 0.6 , at $32 \mathrm{meV}$ for $x=0.8$, and at 34 $\mathrm{meV}$ for $x=0.95$. The solid lines are guides to the eye.

doped compounds, but for highly doped materials they are only found close to the resonance in the superconducting state.

At present, it remains unclear how to explain the observed behavior differences in these two families of materials from a stripe model. In particular, the saturation of the incommensurability at $\delta=1 / 10$ for effective hole concentration $p \geq 0.1$ (Fig. 24) is puzzling. Assuming that the incommensurate spin fluctuations in $\mathrm{YBa}_{2} \mathrm{Cu}_{3} \mathrm{O}_{6+x}$ at all doping levels are due to dynamic stripes composed of the hole-rich metallic regions separated by the antiphase insulating domains [46], one would expect the growth of the metallic stripes with increasing doping at the expense of the insulating domains 69, 70, 71, 72. As a consequence, the incommensurability should increase with increasing hole doping which is in contrast to the observation of a saturating $\delta$ for $\mathrm{YBa}_{2} \mathrm{Cu}_{3} \mathrm{O}_{6+x}$ with $x \geq 0.6$.

Although we have emphasized the differences, the incommensurate spin fluctuations for these two classes of materials also have remarkable similarities in their structure and doping dependence for oxygen concentrations $x \leq 0.6(p \leq 0.1)$. These similarities and the onedimensional nature of the incommensurate spin fluctuations in underdoped $\mathrm{YBa}_{2} \mathrm{Cu}_{3} \mathrm{O}_{6.6}$ [4] and the associ- ated phonon anomaly 85 are consistent with the stripe formation in $\mathrm{YBa}_{2} \mathrm{Cu}_{3} \mathrm{O}_{6+x}$ for effective hole doping below 0.1 . To truly understand the microscopic origin of the incommensurate spin fluctuations in different cuprates using a stripe model, perhaps one needs to first explain the presence of resonance in the bilayer compounds, which takes much of the weight of the total magnetic scattering, and its absence in the single-layer materials [47. For highly doped materials, the incommensurate spin fluctuations in $\mathrm{YBa}_{2} \mathrm{Cu}_{3} \mathrm{O}_{6+x}$ appears to be intimately related to the resonance as they occur only at energies very close to the resonance. From the magnetic field dependence of the spin excitations [68], it is clear that the resonance measures the pairing and long-range phase coherence. Thus, if a stripe model can explain the resonance, it may also be able to account for the differences in the incommensurability in $\mathrm{La}_{2-x} \mathrm{Sr}_{x} \mathrm{CuO}_{4}$ and $\mathrm{YBa}_{2} \mathrm{Cu}_{3} \mathrm{O}_{6+x}$.

Alternatively, assuming that the observed incommensurate fluctuations in $\mathrm{YBa}_{2} \mathrm{Cu}_{3} \mathrm{O}_{6+x}$ come from a nested Fermi surface 81, 82, 83, 84, one may ask whether such models can predict the observed intensity and doping dependent behavior. Unfortunately, there are no explicit predictions about the doping dependence of the incommensurability and its relationship with the resonance from a nested Fermi surface model that can be directly compared with our experiments. Therefore, it is still not clear whether the observed incommensurate fluctuations in $\mathrm{YBa}_{2} \mathrm{Cu}_{3} \mathrm{O}_{6+x}$ are consistent with the Fermi-liquid description at all doping levels. In particular, the abrupt saturation of the incommensurability for $\mathrm{YBa}_{2} \mathrm{Cu}_{3} \mathrm{O}_{6+x}$ with $x \geq 0.6$ suggests that the physics accounting for this behavior may have a different microscopic origin from that of the lower doping compounds.

\section{Models for the resonance}

Since the discovery of the resonance in highly doped and underdoped $\mathrm{YBa}_{2} \mathrm{Cu}_{3} \mathrm{O}_{6+x}$ 12, 14, 16, 17, 18, 19, 20, 21], many fundamentally different microscopic mechanisms have been proposed to explain its origin. In the earlier theoretical studies [86, 87, 88, 89], the resonance is interpreted as the consequence of the $d$-wave gap symmetry of the cuprate superconductors. Subsequent workers have noticed that $d$-wave superconductivity alone is insufficient to explain the resonance, and that very strong Coulomb correlations among electrons must also play an important role. In some models, the band structure anomaly effects alone can produce a peak in spin susceptibility that grows with the opening of the superconducting gap 990, 91, 92], while other workers have applied a random-phase approximation (RPA) to treat the effects of strong Coulomb correlations with the Lindhard function appropriate for either a Fermi liquid $93,94,95$, 96] or a spin-charge separated metal [31, 97, 98]. In particular, the resonance has been treated as a disordered magnon-like collective mode in the particle-hole channel 
whose energy is bound by the superconducting energy gap $82,99,100$. Using a completely different approach [101, the resonance is viewed as a pseudo-Goldstone boson mode in the particle-particle channel instead of the more conventional particle-hole channel. In the framework of this theory, antiferromagnetism and $d$-wave superconductivity in copper oxides are treated in equal footing 102], and resonance is directly responsible for the superconducting condensation energy 61.

While the purpose of this article is not to review the similarities and differences among various models of the resonance, we wish to make several general remarks in light of the results described here and in recent fielddependent studies of the resonance 68. First, it is now clear that the incommensurability and the resonance are inseparable parts of the general features of the spin dynamics in $\mathrm{YBa}_{2} \mathrm{Cu}_{3} \mathrm{O}_{6+x}$ at all dopings. Thus any microscopic model used to describe the resonance must also be able to explain the doping and energy dependence of the incommensurate spin fluctuations. While current theoretical models do not provide explicit predictions that can be directly compared our experiments [103], it is hoped that the work described here will stimulate future theoretical efforts in this direction. The remarkable field dependence of the resonance [68] indicates that it measures the superconducting pairing and phase coherence. In particular, the larger $c$-axis field effect is difficult to understand within the framework of the interlayer tunneling theory, according to which the resonance should be strongly affected by the in-plane field that disrupts the coherent Josephson coupling along $c$ axis [92]. On the other hand, the field data [68 are consistent with mechanism where the dominant loss of entropy on entering the superconducting state is due to the growth of magnetic correlations in the $\mathrm{CuO}_{2}$ planes [60, 61, 62]. Finally, if the resonance is a magnon-like collective mode [82, 99, 100], it must not be a spin-wave in the conventional sense as the intensity of spin-wave excitations in ferromagnets and antiferromagnets should be magnetic field independent for the relatively small fields used [38, 68.

\section{SUMMARY AND CONCLUSIONS}

In this article, we have described in detail the extensive polarized and unpolarized neutron measurements of the dynamical magnetic susceptibility $\chi^{\prime \prime}(\mathbf{q}, \omega)$ of superconducting $\mathrm{YBa}_{2} \mathrm{Cu}_{3} \mathrm{O}_{6+x}$ above and below the transition temperature $T_{c}$. Using a careful polarization and temperature dependence analysis, we find that the resonance and incommensurate spin fluctuations are general features of the spin dynamical behavior in $\mathrm{YBa}_{2} \mathrm{Cu}_{3} \mathrm{O}_{6+x}$ for all oxygen doping levels. We establish the doping de- pendence of the incommensurability, the resonance, and the superconducting spin-gap energy. In the superconducting state, we find that the spin-gap energy is proportional to $k_{B} T_{c}$, but the resonance energy itself deviates from the linear behavior with $k_{B} T_{c}$ for oxygen doping close to the optimal values. Similarly, we show that the doping dependence of the incommensurability saturates to $\delta=0.1$ for oxygen doping $x \geq 0.6$. We compare the observed behaviors in the two most studied superconducting copper-oxides $\mathrm{La}_{2-x} \mathrm{Sr}_{x} \mathrm{CuO}_{4}$ and $\mathrm{YBa}_{2} \mathrm{Cu}_{3} \mathrm{O}_{6+x}$ and discuss their similarities and differences. We stress that any comprehensive theoretical understanding of the spin dynamical behavior in these two families of materials must take into account the fact that the resonance so clearly seen in the bilayer $\mathrm{YBa}_{2} \mathrm{Cu}_{3} \mathrm{O}_{6+x}$ is absent in the single-layer $\mathrm{La}_{2-x} \mathrm{Sr}_{x} \mathrm{CuO}_{4}$. For underdoped $\mathrm{YBa}_{2} \mathrm{Cu}_{3} \mathrm{O}_{6+x}$, the evolution of the resonance as a function of doping is illustrated. In particular, we find the enhancement of the susceptibility at the resonance energy above $T_{c}$ for all underdoped compounds including $\mathrm{YBa}_{2} \mathrm{Cu}_{3} \mathrm{O}_{6.8}$. If we define the "resonance" as the enhancement of the susceptibility in a small energy range of the magnetic excitations spectra as a function of decreasing temperature, it is natural to associate the susceptibility gain around the resonance energy above $T_{c}$ in the underdoped compounds as the precursor of the resonance. This assertion is supported by the systematic studies of the evolution of the temperature dependence of the resonance that show the progressively larger pretransitional regime above $T_{c}$ for more underdoped $\mathrm{YBa}_{2} \mathrm{Cu}_{3} \mathrm{O}_{6+x}$ 27. Furthermore, the resonance and incommensurate fluctuations appear to be intimately connected. Thus, any microscopic model for the magnetic fluctuations in $\mathrm{YBa}_{2} \mathrm{Cu}_{3} \mathrm{O}_{6+x}$ must be able to account for both the incommensurate spin fluctuations and the resonance.

\section{Acknowledgments}

We are grateful to G. Aeppli, S. Chakravarty, T. Egami, V. J. Emery, S. M. Hayden, S. A. Kivelson, D. K. Morr, D. Pines, D. J. Scalapino, J. Zaanen, and S. C. Zhang for many enlightening discussions. We also wish to thank J. A. Fernandez-Baca, Jiandi Zhang, and S. C. Zhang for a critical reading of the manuscript, to thank J. R. Thompson and K. J. Song for measuring the superconducting transition temperatures for all the samples discussed in this article, and to thank B. C. Chakoumakos and T. B. Lindemer for allowing the use of their unpublished data. Finally, we acknowledge much technical support over the years by R. G. Maple, S. A. Moore, G. B. Taylor, and C. M. Redmon. This work was supported by U.S. DOE under contract DE-AC05-00OR22725 with UT-Battelle, LLC. 
[2] J. M. Tranquada, D. E. Cox, W. Kunnmann, H. Moudden, G. Shirane, M. Suenaga, P. Zolliker, D. Vaknin, S. K. Sinha, M. S. Alvarez, A. J. Jacobson, and D. C. Jonhston, Phys. Rev. Lett. 60, 156 (1988).

[3] H. Yoshizawa, S. Mitsuda, H. Kitazawa, and K. Katsumata, J. Phys. Soc. Jpn, 57, 3686 (1988).

[4] R. J. Birgeneau, Y. Endoh, Y. Hidaka, K. Kakurai, M. A. Kastner, T. Murakami, G. Shirane, T. R. Thurston, and K. Yamada, Phys. Rev. B 39, 2868 (1989).

[5] S-W. Cheong, G. Aeppli, T. E. Mason, H. A. Mook, S. M. Hayden, P. C. Canfield, Z. Fisk, K. N. Clausen, and J. L. Martinez, Phys. Rev. Lett. 67, 1791 (1991).

[6] T. E. Mason, G. Aeppli, and H. A. Mook, Phys. Rev. Lett. 68, 1414 (1992).

[7] T. R. Thurston, P. M. Gehring, G. Shirane, R. J. Birgeneau, M. A. Kastner, Y. Endoh, M. Matsuda, K. Yamada, H. Kojima, and I. Tanaka, Phys. Rev. B 46, 9128 (1992).

[8] K. Yamada, C. H. Lee, K. Kurahashi, J. Wada, S. Wakimoto, S. Ueki, H. Kimura, Y. Endoh, S. Hosoya, G. Shirane, R. J. Birgeneau, M. Greven, M. A. Kastner, and Y. J. Kim, Phys. Rev. B 57, 6165 (1998).

[9] K. Yamada, S. Wakimoto, G. Shirane, C. H. Lee, M. A. Kastner, S. Hosoya, M. Greven, Y. Endoh, and R. J. Birgeneau, Phys. Rev. Lett. 75, 1626 (1995).

[10] B. Lake, G. Aeppli, T. E. Mason, A. Schroder, D. F. McMorrow, K. Lefmann, M. Isshiki, M. Nohara, H. Takagi, and S. M. Hayden, Nature (London) 400, 43 (1999).

[11] For a review, see M. A. Kastner, R. J. Birgeneau, G. Shirane, and Y. Endoh, Rev. Mod. Phys. 70, 897 (1998).

[12] J. Rossat-Mignod, L. P. Regnault, C. Vettier, P. Bourges, P. Burlet, J. Bossy, J. Y. Henry, and G. Lapertot, Physica (Amsterdam) 185C, 86 (1991).

[13] J. M. Tranquada, P. M. Gehring, G. Shirane, S. Shamoto, and M. Sato, Phys. Rev. B 46, 5561 (1992).

[14] H. A. Mook, M. Yethiraj, G. Aeppli, T. E. Mason, and T. Armstrong, Phys. Rev. Lett. 70, 3490 (1993).

[15] B. J. Sternlieb, J. M. Tranquada, G. Shirane, M. Sato, and S. Shamoto, Phys. Rev. B 50, 12915 (1994).

[16] H. F. Fong, B. Keimer, P. W. Anderson, D. Reznik, F. Doğan, and I. A. Aksay, Phys. Rev. Lett. 75, 316 (1995).

[17] P. Bourges, L. P. Regnault, Y. Sidis, and C. Vettier, Phys. Rev. B 53, 876 (1996).

[18] H. F. Fong, B. Keimer, D. Reznik, D. M. Milius, and I. A. Aksay, Phys. Rev. B 546708 (1996).

[19] P. Dai, M. Yethiraj, H. A. Mook, T. B. Lindemer, and F. Doğan, Phys. Rev. Lett. 775425 (1996).

[20] H. F. Fong, B. Keimer, F. Doğan, and I. A. Aksay, Phys. Rev. Lett. 78, 713 (1997).

[21] P. Bourges, H. F. Fong, L. P. Regnault, J. Bossy, C. Vettier, D. L. Milius, I. A. Aksay, and B. Keimer, Phys. Rev. B 56, R11439 (1997).

[22] P. Dai, H. A. Mook, and F. Doğan, Phys. Rev. Lett. 80, 1738 (1998).

[23] H. A. Mook, P. Dai, R. D. Hunt, and F. Doğan, J. Phys. Chem. Solids 59, 2140 (1998).

[24] H. A. Mook, P. Dai, S. M. Hayden, G. Aeppli, T. G. Perring, and F. Doğan, Nature (London) 395, 580 (1998).

[25] M. Arai, T. Nishijima, Y. Endoh, T. Egami, S. Tajima, K. Tomimoto, Y. Shiohara, M. Takahashi, A. Garret, and S. M. Bennington, Phys. Rev. Lett. 83, 608 (1999).

[26] S. M. Hayden, G. Aeppli, P. Dai, H. A. Mook, T. G. Perring, S.-W. Cheong, Z. Fisk, F. Doğan, and T. E.
Mason. Physica B 241-243, 765 (1998).

[27] P. Dai, H. A. Mook, S. M. Hayden, G. Aeppli, T. G. Perring, R. D. Hunt, and F. Doğan, Science 284, 1344 (1999).

[28] D. J. Scalapino, Science 284, 1282 (1999).

[29] To facilitate the comparison of the results between $\mathrm{La}_{2-x} \mathrm{Sr}_{x} \mathrm{CuO}_{4}$ and $\mathrm{YBa}_{2} \mathrm{Cu}_{3} \mathrm{O}_{6+x}$, we adopted the definition of incommensurability $\delta$ used in [9]. Note that this definition of incommensurability $\delta$ is different (by a factor of 2) from that in [24].

[30] C. Michel and B. Raveau, J. Solid State Chem. 43, 73 (1982).

[31] R. Gopalan, T. Rajasekharan, T. Roy, G. Rangarajan, V. Ganesan, and R. Srinivasan, J. Mater. Res. 11, 2406 (1996).

[32] T. B. Lindemer, (unpublished).

[33] T. B. Lindemer, J. F. Hunley, J. E. Gates, A. L. Sutton, J. Brynestard, C. R. Hubbard, P. K. Gallagher, J. Am. Ceram. Soc. 72, 1775 (1989).

[34] P. Dai, J. Zhang, H. A. Mook, S.-H. Liou, P. A. Dowben, and E. W. Plummer, Phys. Rev. B 54, R3694 (1996).

[35] J. D. Jorgensen, B. W. Veal, A. P. Paulikas, L. J. Nowicki, G. W. Grabtree, H. Claus, and W. K. Kwok, Phys. Rev. B 41, 1863 (1990).

[36] E. Altendorf, X. K. Chen, J. C. Irwin, R. Liang, and W. N. Hardy, Phys. Rev. B 47, 8140 (1993).

[37] B. C. Chakoumakos and T. B. Lindermer, (unpulished work).

[38] S. W. Lovesey, in Theory of thermal neutron scattering from condensed matter (Clarendon, Oxford, 1984), Vol. 2.

[39] R. M. Moon, T. Riste, and W. C. Koehler, Phys. Rev. 181, 920 (1969).

[40] Strictly speaking, this equation is only true if the efficiency of the Mezei coil is $100 \%$.

[41] S. M. Hayden, G. Aeppli, T. G. Perring, H. A. Mook, F. Doğan, Phys. Rev. B 54, R6905 (1996)

[42] D. Reznik, P. Bourges, H. F. Fong, L. P. Regnault, J. Bossy, C. Vettier, D. L. Milius, I. A. Aksay, and B. Keimer, Phys. Rev. B 53, R14741 (1996).

[43] A. V. Balatsky and P. Bourges, Phys. Rev. Lett. 82, 5337 (1999).

[44] Although there is also a spin pseudogap in the normal state of $\mathrm{YBa}_{2} \mathrm{Cu}_{3} \mathrm{O}_{6+x}$, we did not systematically investigate its temperature and doping dependence because the difficulty in separating the low-energy magnetic scattering from phonons and other spurious processes.

[45] J. L. Tallon, C. Bernhard, H. Shaked, R. L. Hitterman, and J. D. Jorgensen, Phys. Rev. B 51, 12911 (1995).

[46] J. M. Tranquada, B. J. Sternlieb, J. D. Axe, Y. Nakamura, and S. Uchida, Nature (London) 375, 561 (1995); J. M. Tranquada, J. D. Axe, N. Ichikawa, Y. Nakamura, S. Uchida, and B. Nachumi, Phys. Rev. B 54, 7489 (1996).

[47] H. A. Mook, P. Dai, F. Doğan, and R. D. Hunt, Nature (London) 404, 729 (2000).

[48] Although one expects incommensurate spin fluctuations at this doping level, detection of explicit incommensurability may require samples to have single grain with narrow mosaic spread. The mosaic of $\mathrm{YBa}_{2} \mathrm{Cu}_{3} \mathrm{O}_{6.5}$ is considerably larger than other crystals in this study. As a consequence, we only an observe broad flattish top peak around $(\pi, \pi)$ at $\hbar \omega=24 \mathrm{meV}$ [Fig. 8(c)]. The in- 
commensurability was determined by fitting the $24 \mathrm{meV}$ profile with a pair of Gaussian peaks separated by $\delta$ rlu from $(\pi, \pi)$.

[49] G. Aeppli, T. E. Mason, S. M. Hayden, H. A. Mook, and J. Kulda, Science 278, 1432 (1997).

[50] Although we have demonstrated the clear presence incommensurate spin fluctuations for $\mathrm{YBa}_{2} \mathrm{Cu}_{3} \mathrm{O}_{6+x}$ with $x=0.45,0.6,0.7,0.8$, and 0.95, no explicit incommensurability is observed for our $\mathrm{YBa}_{2} \mathrm{Cu}_{3} \mathrm{O}_{6.5}$ sample and the clarity of incommensurate spin fluctuations appears to be sample dependent. At present, we do not understand the microscopic origin for such behavior. Perhaps the detailed incommensurate structure is very sensitive to the oxygen uniformity of the material.

[51] P. Bourges, Y. Sidis, H. F. Fong, L. P. Regnault, J. Bossy, A. Ivanov, and B. Keimer, Science 288, 1234 (2000).

[52] S. Uchida, T. Ido, H. Takai, T. Arima, Y. Tokura, and S. Tajima, Phys. Rev. B 43, 7942 (1991).

[53] T. Ito, K. Takenaka, S. Uchida, Phys. Rev. Lett. 70, 3995 (1993).

[54] B. Wuyts, V. V. Moshchalkov, Y. Bruynseraede, Phys. Rev. B 53, 9418 (1996).

[55] M. Takigawa, A. P. Reyes, P. C. Hammel, J. D. Thompson, R. H. Heffner, Z. Fisk, and K. C. Ott, Phys. Rev. B 43, 247 (1991); H. Alloul, A. Mahajan, H. Casalta, and O. Klein, Phys. Rev. Lett. 70, 1171 (1993); M. Horvatić, T. Auler, C. Berthier, Y. Berthier, P. Butaud, W. G. Clark, J. A. Gillet, P. Ségransan, and J. Y. Henry, Phys. Rev. B 47, 3461 (1993).

[56] H. F. Fong, P. Bourges, Y. Sidis, L. P. Regnault, J. Bossy, A. Ivanov, D. L. Milius, I. A. Aksay, and B. Keimer, Phys. Rev. B 61, 14733 (2000).

[57] P. Bourges, B. Keimer, L. P. Regnault, and Y. Sidis, cond-mat/0006085 (unpublished).

[58] P. Bourges, in The gap Symmetry and Fluctuations in High Temperature Superconductors, Edited by J. Bok, G. Deutscher, D. Pavuna, and S. A. Wolf (Plenum Press, 1998) p349.

[59] J. W. Loram, K. A. Mirza, J. R. Cooper, and W. Y. Liang, Phys. Rev. Lett. 71, 1740 (1993).

[60] D. J. Scalapino and S. R. White, Phys. Rev. B 58, 8222 (1998).

[61] E. Demler and S. C. Zhang, Nature (London) 396, 733 (1998).

[62] S. Chakravarty and H. Y. Kee, Phys. Rev. B 61, 14821 (2000).

[63] M. R. Norman, M. Randeria, B. Jankó, and J. C. Campuzano, Phys. Rev. B 61, 14742 (2000).

[64] B. Jankó, cond-mat/9912073 (unpublished).

[65] J. R. Shrieffer, Theory of Superconductivity, (Benjamin, New York, 1964).

[66] A. Junod, in Studies of high temperature superconductors, Vol. 19, Ed. A. V. Narlikar, Nova Science Publishers, N. Y. 1996, page 1.

[67] A. Junod, A. Erb, and Ch. Renner, Physica C 317, 333 (1999).

[68] P. Dai, H. A. Mook, G. Aeppli, S. M. Hayden, and F. Doğan, Nature (London) 406, 965 (2000).

[69] J. Zaanen and O. Gunnarson, Phys. Rev. B 40, 7391 (1989).

[70] H. J. Schulz, J. Phys. (Paris) 50, 2833 (1989).

[71] D. Poilblanc, and T. M. Rice, Phys. Rev. B 39, 9749 (1989).
[72] S. A. Kivelson and V. J. Emery, in Strongly correlated electronic materials: the Los Alamos symposium 1993 (eds K. S. Bedell et al., Addison-Wesley, Maine, 1994), p 619.

[73] For a recent review on advances in the physics of high$T_{c}$ superconductivity, see J. Orenstein and A. J. Millis, Science 288, 468 (2000).

[74] J. Zaanen, J. Phys. Chem. Solids 59, 1769 (1998).

[75] V. J. Emery, S. A. Kivelson, and O. Zahar, Phys. Rev. B 56, 6120 (1997).

[76] S. R. White and D. J. Scalapino, Phys. Rev. Lett. 80, 1272 (1998).

[77] G. B. Martins, C. Gazza, J. C. Xavier, A. Feiguin, and E. Dagotto, Phys. Rev. Lett. 84, 5844 (2000).

[78] B. Bulut, D. Hone, D. J. Scalapino, and N. E. Bickers, Phys. Rev. Lett. 64, 2466 (1990).

[79] Q. M. Si, Y. Zha, K. Levin, and J. P. Lu, Phys. Rev. B 47, 9055 (1993); Y. Zha, K. Levin, and Q. M. Si, Phys. Rev. B 47, 9124 (1993).

[80] P. B. Littlewood, J. Zaanen, G. Aeppli, and H. Monien, Phys. Rev. B 48, 487 (1993).

[81] J. Brinckmann, and P. A. Lee, Phys. Rev. Lett. 82, 2915 (1999).

[82] D. K. Morr and D. Pines, Phys. Rev. Lett. 81, 1086 (1998); Phys. Rev. B 61, 6483 (2000).

[83] Y. J. Kao, Q. Si, and K. Levin, Phys. Rev. B 61, 11898 (2000).

[84] M. R. Norman, Phys. Rev. B 61, 14751 (2000).

[85] H. A. Mook and F. Doğan, Nature (London) 401, 145 (1999).

[86] K. Maki and H. Won, Phys. Rev. Lett. 72, 1758 (1994).

[87] M. J. Lercher and J. M. Weatley, Phys. Rev. B 49, 736 (1994).

[88] M. Lavagna and G. Stemmann, Phys. Rev. B 49, 4235 (1994); G. Stemmann, C. Pépin, and M. Lavagna, Phys. Rev. B 50, 4075 (1994).

[89] F. Onufrieva and J. Rossat-Mignod, Phys. Rev. B 52, 7572 (1995).

[90] G. Blumberg, B. P. Stojkovic, and M. V. Klein, Phys. Rev. B 52, 15741 (1995).

[91] A. A. Abrikosov, Phys. Rev. B 57, 8656 (1998).

[92] Lan Yin, S. Chakravarty, and P. W. Anderson, Phys. Rev. Lett. 78, 3559 (1997).

[93] N. Bulut and D. J. Scalapino, Phys. Rev. B 53, 5149 (1996).

[94] D. Z. Liu, Y. Zha, and K. Levin, Phys. Rev. Lett. 75, 4130 (1995)

[95] I. I. Mazin and V. M. Yakovenko, Phys. Rev. Lett.75, 4134 (1995).

[96] A. J. Millis and H. Monien, Phys. Rev. B 54, 16172 (1996).

[97] T. Tanamoto, H. Kohno, and H. Fukuyama, J. Phys. Soc. Jpn. 611886 (1992).

[98] B. Normand, H. Kohno, and H. Fukuyama, J. Phys. Jpn. 64, 3903 (1995)

[99] A. Abanov and A. V. Chubukov, Phys. Rev. Lett. 83, 1652 (1999).

[100] S. Sachdev, C. Buragohain, and M. Vojta, Science 286, 2479 (1999).

[101] E. Demler and S. C. Zhang, Phys. Rev. Lett. 75, 4126 (1995); E. Demler, H. Kohno, and S. C. Zhang, Phys. Rev. B 58, 5719 (1998).

[102] S. C. Zhang, Science 275, 1089 (1997).

[103] After the completion of this article, we noted a theo- 
retical attempt to reconcile the differences between spin fluctuations in $\mathrm{La}_{2-x} \mathrm{Sr}_{x} \mathrm{CuO}_{4}$ and $\mathrm{YBa}_{2} \mathrm{Cu}_{3} \mathrm{O}_{6+x}$. K.

(unpublished). 